\title{
Inchworm Monte Carlo method for open quantum systems
}

\author{
Zhenning $\mathrm{Cai}^{1}$, Jianfeng $\mathrm{Lu}^{2}$, Siyao Yang ${ }^{1}$
}

\begin{abstract}
We investigate in this work a recently proposed diagrammatic quantum Monte Carlo method — the inchworm Monte Carlo method - for open quantum systems. We establish its validity rigorously based on resummation of Dyson series. Moreover, we introduce an integro-differential equation formulation for open quantum systems, which illuminates the mathematical structure of the inchworm algorithm. This new formulation leads to an improvement of the inchworm algorithm by introducing classical deterministic time-integration schemes. The numerical method is validated by applications to the spin-boson model.
\end{abstract}

Keywords: quantum Monte Carlo, open quantum system, diagrammatic methods, spin-boson model

\section{Introduction}

For realistic quantum systems, the system we are interested in is often coupled with an uninteresting environment to a non-negligible extent, which requires us to study the open quantum system, including the effects such as quantum decoherence [57] and quantum dissipation [18]. The application of open quantum system ranges in a wide variety of quantum fields, including quantum optical systems [3], nonlinear statistical mechanics [34], quantum computation [47], etc. In reality, no quantum system in experiment is completely isolated from the environment. The development of quantum technology also relies on mitigating and sometimes utilizing the quantum dissipation [48], which calls for better understanding of open quantum systems both theoretically and numerically. The open quantum system can also arise from approximate algorithms for many-body quantum systems in condensed matter physics, such as the rather successful dynamical mean field theory (DMFT) approach which reduces a full many-body system to an impurity problem which is then treated numerically as an open quantum system [23].

The challenge of numerical simulation of open quantum system lies in the coupling of the system of interest to a usually large bath which contains a huge number of degree of freedom. This makes the direct simulation of the whole system together with bath impossible. Thus it is necessary to carry out dimension reduction, hopefully to integrate out the degree of freedoms associated with the bath. Such issues of dimension reduction also arise in many other areas of modeling and simulations. Indeed, while we focus in this work on open quantum system, many of the ideas have their classical counterparts, some of them have led to active developments in applied mathematics, see e.g., [6, 8, , 9, 11, 25, 31, 32, 46, ,58] and books [7, 17, 19, 20, 27]. It is our hope that study of dimension reduction for open quantum systems can also shed light on further development for dimension reduction of classical systems.

In the context of open quantum system, many dimension reduction techniques have been developed over the years. One conventional approach is to apply the Nakajima-Zwanzig projection operator technique to obtain an integro-differential master equation [45, 61], where the memory effects resulting from integrating out the bath degree of freedom are encoded by some memory kernel. The classical analog is the well-known Mori-Zwanzig formalism [44, 62]. By taking the weak-coupling limit, the memory effect can be neglected

\footnotetext{
${ }^{1}$ Department of Mathematics, National University of Singapore, Level 4, Block S17, 10 Lower Kent Ridge Road, Singapore 119076.

${ }^{2}$ Department of Mathematics, Department of Physics, Department of Chemistry, Duke University, Box 90320, Durham NC 27708, USA.
} 
to obtain a Markovian approximation, known as the Lindblad equation 12, 13], which is the quantum analog of the Langevin dynamics. The Markovian approximation however breaks down for open systems with stronger coupling, so that one has to keep track of the non-Markovian dynamics of the projected density matrix describing the system of interests 3]. For classical systems, such dynamics is often modeled and studied as generalized Langevin equations [24, 63], while for quantum systems, the non-Markovian dynamics is much more complicated, and many works have been devoted to this topic. Analogously to the generalized Langevin equations, generalized quantum master equation with memory kernel has been used to model non-Markovian open quantum dynamics [29, 42, 43, 52], though it is often complicated to come up with a good estimate of the memory kernel, especially when the memory is long range.

Besides the quantum master equation framework, the non-Markovian evolution of the system can be also directly modeled and simulated using the path-integral approaches such as the QuAPI (quasi-adiabatic propagator path integral) methods [36, 41] and the HEOM (hierarchical equations of motion) technique [56]. These methods yield accurate numerical results (often referred as "numerically exact" in the literature), while the computational cost is extremely huge, often unaffordable. To reduce the computational cost, one common strategy is to replace the exact summation or numerical integration in these methods by Monte Carlo methods. In this paper, we are going to study a specific type of path-integral methods called the diagrammatic quantum Monte Carlo method [60] to solve the time-dependent open quantum systems. In particular, our study is largely motivated by the inchworm Monte Carlo method recently proposed in [4, 10] to reduce the variance in quantum Monte Carlo by diagrammatic resummation.

The basis of the diagrammatic quantum Monte Carlo method has been established as early as 1960s 28]. However, as other quantum Monte Carlo methods, this type of methods also suffer from the notorious dynamical sign problem, meaning that the number of Monte Carlo samples is required to grow at least exponentially in time in order to keep the accuracy of the simulation. To relieve the dynamical sign problem, Stockburger and Grabert introduced stochastic unraveling of influence functionals in [55], and Makri [35, 38] proposed to assume a finite memory time of the bath-correlation function and apply an iterative procedure to efficiently implement the summation. The inchworm Monte Carlo method applies the idea of diagrammatic resummation as in the bold diagrammatic Monte Carlo method [49] to the real-time evolution of the quantum systems. Similar to the bold-line diagrammatic Monte Carlo method proposed in 21, 22] (see also a more mathematical presentation [33]), the inchworm Monte Carlo method tackles the dynamical sign problem by lumping a large number of diagrams into a "bold line", which effectively reduces the number of total diagrams to be summed to reach desired numerical accuracy. The inchworm method makes maximum use of the previous calculations, at the expense of higher memory cost for storing all Green's functions. Despite its success in the application of spin-boson model [5] and the Anderson impurity model [14, [50], it requires better understanding to reveal the intrinsic mathematical structure of the inchworm method, and to further improve the method. In particular, it would be interesting to see how the bold lines are built on the basis of shorter bold lines, and how the bold lines propagate when the iterative procedure is precise. While the answers to these questions are not detailed in the original derivation of the inchworm method [4, 10], in this paper, we will show the validity of the inchworm method with mathematical rigor. The rigorous proof not only justifies the original algorithm, but also leads us to a new formulation of the open quantum system as an integro-differential equation, based on which more accurate and efficient numerical approaches can be developed.

The inchworm Monte Carlo method and the new integro-differential equation formulation will be proved to be applicable for the Ohmic spin-boson model, which is a simple open quantum system widely used as benchmark problems [15, 30, 59]. Based on the integro-differential equation, part of the Monte Carlo integration can be replaced by classical time-integration methods to achieve higher accuracy. The resulting new algorithm will be applied to the spin-boson model to show the numerical efficiency. Possible extensions will also be discussed. More generally, the inchworm method represents a wider class of model reduction and variance reduction techniques for open quantum systems, which are worth investigating from the mathematical point of view, as they offer some new challenges as well as new toolsets that might be applicable to other areas of study.

The rest of this paper is organized as follows. In Section 2, we introduce the basic formulation of the open quantum system and its Dyson series expansion. Section 3 gives a complete review of the inchworm 
Monte Carlo method and proves its validity. The integro-differential equation associate with the inchworm algorithm is derived in Section 4. As an application, we analyze the spin-boson model in Section 5 . Our new numerical method is introduced in Section 6 and some numerical examples are given in Section 7 . A simple summary is given in Section 8 as the end of the paper.

\section{Dyson series expansion for open quantum systems}

Before considering open quantum system, let us first recall the time-dependent perturbation theory and the associated Dyson series. Consider the von Neumann equation for quantum evolution (of a closed system)

$$
\mathrm{i} \frac{\mathrm{d} \rho}{\mathrm{d} t}=[H, \rho]
$$

where $\rho(t)$ is the density matrix at time $t$, and $H$ is the Schrödinger picture Hamiltonian with the form

$$
H=H_{0}+W \text {. }
$$

Here $H_{0}$ is the unperturbed Hamiltonian and $W$ is viewed as a perturbation. Following the convention, for any Hermitian operator $A$, we define $\langle A\rangle=\operatorname{tr}(\rho(0) A)$. We are interested in the evolution of the expectation for a given observable $O$, defined by

$$
\langle O(t)\rangle=\operatorname{tr}(O \rho(t))=\operatorname{tr}\left(O \mathrm{e}^{-\mathrm{i} t H} \rho(0) \mathrm{e}^{\mathrm{i} t H}\right)=\left\langle\mathrm{e}^{\mathrm{i} t H} O \mathrm{e}^{-\mathrm{i} t H}\right\rangle .
$$

Using standard time dependent perturbation theory, the unitary group $\mathrm{e}^{-\mathrm{i} t H}$ generated by $H$ can be represented using a Dyson series expansion [16]

$$
\mathrm{e}^{-\mathrm{i} t H}=\sum_{n=0}^{+\infty} \int_{t>t_{n}>\cdots>t_{1}>0}(-\mathrm{i})^{n} \mathrm{e}^{-\mathrm{i}\left(t-t_{n}\right) H_{0}} W \mathrm{e}^{-\mathrm{i}\left(t_{n}-t_{n-1}\right) H_{0}} W \cdots W \mathrm{e}^{-\mathrm{i}\left(t_{2}-t_{1}\right) H_{0}} W \mathrm{e}^{-\mathrm{i} t_{1} H_{0}} \mathrm{~d} t_{1} \cdots \mathrm{d} t_{n},
$$

where the integral should be interpreted as

$$
\int_{t>t_{n}>\cdots>t_{1}>0} \mathrm{~d} t_{1} \cdots \mathrm{d} t_{n}=\int_{0}^{t} \int_{0}^{t_{n}} \cdots \int_{0}^{t_{2}} \mathrm{~d} t_{1} \cdots \mathrm{d} t_{n-1} \mathrm{~d} t_{n}
$$

Inserting the Dyson series (3) into (2), one obtains

$$
\begin{aligned}
\langle O(t)\rangle= & \sum_{n=0}^{+\infty} \sum_{n^{\prime}=0}^{+\infty} \int_{t>t_{n}>\cdots>t_{1}>0} \int_{t>t_{n^{\prime}}^{\prime}>\cdots>t_{1}^{\prime}>0}(-\mathrm{i})^{n} \mathrm{i}^{n^{\prime}} \\
& \left\langle\mathrm{e}^{\mathrm{i} t_{1}^{\prime} H_{0}} W \mathrm{e}^{\mathrm{i}\left(t_{2}^{\prime}-t_{1}^{\prime}\right) H_{0}} W \cdots W \mathrm{e}^{\mathrm{i}\left(t_{n^{\prime}}^{\prime}-t_{n^{\prime}-1}^{\prime}\right) H_{0}} W \mathrm{e}^{\mathrm{i}\left(t-t_{n^{\prime}}^{\prime}\right) H_{0}} O \times\right. \\
& \left.\mathrm{e}^{-\mathrm{i}\left(t-t_{n}\right) H_{0}} W \mathrm{e}^{-\mathrm{i}\left(t_{n}-t_{n-1}\right) H_{0}} W \cdots W \mathrm{e}^{-\mathrm{i}\left(t_{2}-t_{1}\right) H_{0}} W \mathrm{e}^{-\mathrm{i} t_{1} H_{0}}\right\rangle \mathrm{d} t_{1}^{\prime} \cdots \mathrm{d} t_{n}^{\prime} \mathrm{d} t_{1} \cdots \mathrm{d} t_{n} .
\end{aligned}
$$

Since the unperturbed Hamiltonian $H_{0}$ is usually easier to solve, the above expansion provides the basis of a feasible approach to find $\langle O(t)\rangle$ using Monte Carlo method.

For notational simplicity, the above integral is often denoted by the Keldysh contour plotted in Figure 1. The Keldysh contour should be read following the arrows in the diagram, and therefore has a forward (upper) branch and a backward (lower) branch. The symbols are interpreted as follows:

- Each line segment connecting two adjacent time points labeled by $t_{\mathrm{s}}$ and $t_{\mathrm{f}}$ means a propagator $\mathrm{e}^{-\mathrm{i}\left(t_{\mathrm{f}}-t_{\mathrm{s}}\right) H_{0}}$. On the forward branch, $t_{\mathrm{f}}>t_{\mathrm{s}}$, while on the backward branch, $t_{\mathrm{f}}<t_{\mathrm{s}}$.

- Each black dot introduces a perturbation operator $\pm \mathrm{i} W$, where we take the minus sign on the forward branch, and the plus sign on the backward branch. At the same time, every black dot also represents an integral with respect to the label, whose range is from 0 to the adjacent label to its right. 
- The cross sign at time $t$ means the observable in the Schrödinger picture.

Note that according to the above interpretation, two Keldysh contours differ only when at least one of the values of $n, n^{\prime}$ and $t$ is different, while the positions of the labels on each branch do not matter. Thus, by taking the expectation $\langle\cdot\rangle$ of this "contour", we obtain the summand in (5). Therefore $\langle O(t)\rangle$ can be understood as the sum of the expectations of all possible Keldysh contours.

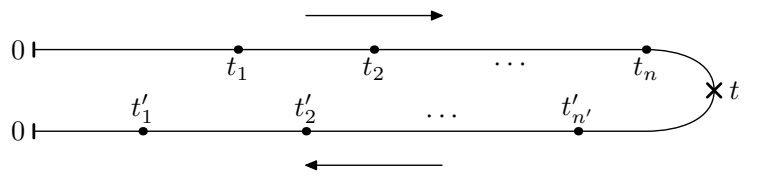

Figure 1: Keldysh contour

Such an interpretation also shows that we do not need to distinguish the forward and backward branches when writing down the integrals. In fact, when the series (5) is absolutely convergent in the sense that

$$
\begin{aligned}
\sum_{n=0}^{+\infty} \sum_{n^{\prime}=0}^{+\infty} & \int_{t>t_{n}>\cdots>t_{1}>0} \int_{t>t_{n^{\prime}}^{\prime}>\cdots>t_{1}^{\prime}>0} \mid\left\langle\mathrm{e}^{\mathrm{i} t_{1}^{\prime} H_{0}} W \mathrm{e}^{\mathrm{i}\left(t_{2}^{\prime}-t_{1}^{\prime}\right) H_{0}} W \cdots W \mathrm{e}^{\mathrm{i}\left(t_{n^{\prime}}^{\prime}-t_{n^{\prime}-1}^{\prime}\right) H_{0}} W \mathrm{e}^{\mathrm{i}\left(t-t_{n^{\prime}}^{\prime}\right) H_{0}} O \times\right. \\
& \left.\times \mathrm{e}^{-\mathrm{i}\left(t-t_{n}\right) H_{0}} W \mathrm{e}^{-\mathrm{i}\left(t_{n}-t_{n-1}\right) H_{0}} W \cdots W \mathrm{e}^{-\mathrm{i}\left(t_{2}-t_{1}\right) H_{0}} W \mathrm{e}^{-\mathrm{i} t_{1} H_{0}}\right\rangle \mid \mathrm{d} t_{1}^{\prime} \cdots \mathrm{d} t_{n}^{\prime} \mathrm{d} t_{1} \cdots \mathrm{d} t_{n}<+\infty
\end{aligned}
$$

we can reformulate (5) as

$$
\begin{aligned}
\langle O(t)\rangle= & \sum_{m=0}^{+\infty} \int_{2 t>s_{m}>\cdots>s_{1}>0}(-1)^{\#\{s<t\}_{1}{ }^{m}} \times \\
& \times\left\langle G^{(0)}\left(2 t, s_{m}\right) W G^{(0)}\left(s_{m}, s_{m-1}\right) W \cdots W G^{(0)}\left(s_{2}, s_{1}\right) W G^{(0)}\left(s_{1}, 0\right)\right\rangle \mathrm{d} s_{1} \cdots \mathrm{d} s_{m},
\end{aligned}
$$

where we use $s$ as a short-hand for the decreasing sequence $\left(s_{m}, \cdots, s_{1}\right)$ and use $\#\{s<t\}$ to denote the number of elements in $s$ which are less than $t$, i.e., the number of $s_{k}$ on the forward branch of the Keldysh contour. For a given $t$, the propagator $G^{(0)}$ is defined as

$$
G^{(0)}\left(s_{\mathrm{f}}, s_{\mathrm{i}}\right)= \begin{cases}\mathrm{e}^{-\mathrm{i}\left(s_{\mathrm{f}}-s_{\mathrm{i}}\right) H_{0}}, & \text { if } s_{\mathrm{i}} \leqslant s_{\mathrm{f}}<t, \\ \mathrm{e}^{\mathrm{i}\left(s_{\mathrm{f}}-s_{\mathrm{i}}\right) H_{0}}, & \text { if } t \leqslant s_{\mathrm{i}} \leqslant s_{\mathrm{f}}, \\ \mathrm{e}^{\mathrm{i}\left(s_{\mathrm{f}}-t\right) H_{0}} O \mathrm{e}^{-\mathrm{i}\left(t-s_{\mathrm{i}}\right) H_{0}}, & \text { if } s_{\mathrm{i}}<t \leqslant s_{\mathrm{f}} .\end{cases}
$$

The integral (6) can also be understood diagramatically as the "unfolded Keldysh contour" plotted in Figure 2. In order to use only a single integral in (6), we set the range of the unfolded Keldysh contour to be [0,2t], and the mapping of time points from the unfolded Keldysh contour to the original Keldysh contour has been implied in the definition of $G^{(0)}(\cdot, \cdot)$. By comparing (6) with Figure 2, one can see that $G^{(0)}(\cdot, \cdot)$ can be considered as the unperturbed propagator on the unfolded Keldysh contour, with an action of observable $O$ at time $t$.

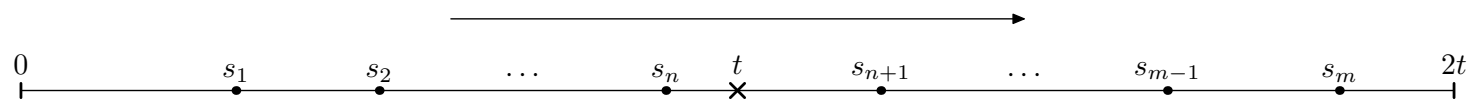

Figure 2: Unfolded Keldysh contour

To proceed, we now assume that the von Neumann equation (11) describes an open quantum system coupled with a bath, which means that both $\rho$ and $H$ are Hermitian operators on the Hilbert space $\mathcal{H}=$ $\mathcal{H}_{s} \otimes \mathcal{H}_{b}$, with $\mathcal{H}_{s}$ and $\mathcal{H}_{b}$ representing respectively the Hilbert spaces associated with the system and the bath. We let $H_{0}$ be the Hamiltonian without coupling:

$$
H_{0}=H_{s} \otimes \operatorname{Id}_{b}+\operatorname{Id}_{s} \otimes H_{b},
$$


where $H_{s}$ and $H_{b}$ are respectively the uncoupled Hamiltonians for the system and the bath, and $\operatorname{Id}_{s}$ and $\operatorname{Id}_{b}$ are respectively the identity operators for the system and the bath. Then the perturbation $W$ describes the coupling, and here we assume $W$ takes the form without loss of generality

$$
W=W_{s} \otimes W_{b} .
$$

Furthermore, we assume the initial density matrix has the separable form $\rho(0)=\rho_{s} \otimes \rho_{b}$, and we are concerned with observables acting only on the system $O=O_{s} \otimes \operatorname{Id}_{b}$ (recall that physically the system is the interesting part). With these assumptions, (6) becomes

$$
\langle O(t)\rangle=\sum_{m=0}^{+\infty} \mathrm{i}^{m} \int_{2 t>s_{m}>\cdots>s_{1}>0}(-1)^{\#\{\boldsymbol{s}<t\}} \operatorname{tr}_{s}\left(\rho_{s} \mathcal{U}^{(0)}(2 t, \boldsymbol{s}, 0)\right) \mathcal{L}_{b}(\boldsymbol{s}) \mathrm{d} s_{1} \cdots \mathrm{d} s_{m},
$$

where the integrand is separated into $\mathcal{U}^{(0)}$ and $\mathcal{L}_{b}$ for the system and bath parts:

$$
\begin{aligned}
\mathcal{U}^{(0)}\left(s_{\mathrm{f}}, \boldsymbol{s}, s_{\mathrm{i}}\right) & =\mathcal{U}^{(0)}\left(s_{\mathrm{f}}, s_{m}, \cdots, s_{1}, s_{\mathrm{i}}\right) \\
& =G_{s}^{(0)}\left(s_{\mathrm{f}}, s_{m}\right) W_{s} G_{s}^{(0)}\left(s_{m}, s_{m-1}\right) W_{s} \cdots W_{s} G_{s}^{(0)}\left(s_{2}, s_{1}\right) W_{s} G_{s}^{(0)}\left(s_{1}, s_{\mathrm{i}}\right), \\
\mathcal{L}_{b}(\boldsymbol{s}) & =\operatorname{tr}_{b}\left(\rho_{b} G_{b}^{(0)}\left(2 t, s_{m}\right) W_{b} G_{b}^{(0)}\left(s_{m}, s_{m-1}\right) W_{b} \cdots W_{b} G_{b}^{(0)}\left(s_{2}, s_{1}\right) W_{b} G_{b}^{(0)}\left(s_{1}, 0\right)\right),
\end{aligned}
$$

where $\operatorname{tr}_{s}$ and $\operatorname{tr}_{b}$ take traces of the system and bath respectively. The propagators $G_{s}^{(0)}$ and $G_{b}^{(0)}$ are defined similarly to (7):

$$
G_{s}^{(0)}\left(s_{\mathrm{f}}, s_{\mathrm{i}}\right)= \begin{cases}\mathrm{e}^{-\mathrm{i}\left(s_{\mathrm{f}}-s_{\mathrm{i}}\right) H_{s}}, & \text { if } s_{\mathrm{i}} \leqslant s_{\mathrm{f}}<t, \\ \mathrm{e}^{-\mathrm{i}\left(s_{\mathrm{i}}-s_{\mathrm{f}}\right) H_{s}}, & \text { if } t \leqslant s_{\mathrm{i}} \leqslant s_{\mathrm{f}}, \\ \mathrm{e}^{-\mathrm{i}\left(t-s_{\mathrm{f}}\right) H_{s}} O_{s} \mathrm{e}^{-\mathrm{i}\left(t-s_{\mathrm{i}}\right) H_{s}}, & \text { if } s_{\mathrm{i}}<t \leqslant s_{\mathrm{f}},\end{cases}
$$

and

$$
G_{b}^{(0)}\left(s_{\mathrm{f}}, s_{\mathrm{i}}\right)= \begin{cases}\mathrm{e}^{-\mathrm{i}\left(s_{\mathrm{f}}-s_{\mathrm{i}}\right) H_{b}}, & \text { if } s_{\mathrm{i}} \leqslant s_{\mathrm{f}}<t, \\ \mathrm{e}^{-\mathrm{i}\left(s_{\mathrm{i}}-s_{\mathrm{f}}\right) H_{b}}, & \text { if } t \leqslant s_{\mathrm{i}} \leqslant s_{\mathrm{f}} \\ \mathrm{e}^{-\mathrm{i}\left(2 t-s_{\mathrm{i}}-s_{\mathrm{f}}\right) H_{b}}, & \text { if } s_{\mathrm{i}}<t \leqslant s_{\mathrm{f}}\end{cases}
$$

Note that the observable $O_{s}$ is inserted into the propagator $G_{s}^{(0)}$ to keep the expression in (9) concise.

\section{Inchworm algorithm}

In this section, we are going to study the inchworm algorithm introduced in [4], where the method was proposed for the spin-Boson model from a purely diagrammatic point of view. By matching the mathematical interpretation and the diagrammatic interpretation of the algorithm, we will establish rigorously the validity of the algorithm in a more general sense. The central idea of the algorithm is to consider the problem as an evolution problem and reuse as much previous information as possible. We will start the introduction of the algorithm by introducing the "full propagators", which are exactly the carriers of the information to be recycled.

\subsection{Full propagator and its Dyson series expansion}

The inchworm algorithm proposed in [4] considers the following "full propagators":

$$
G\left(s_{\mathrm{f}}, s_{\mathrm{i}}\right)= \begin{cases}\operatorname{tr}_{b}\left(\rho_{b} G_{b}^{(0)}\left(2 t, s_{\mathrm{f}}\right) \mathrm{e}^{-\mathrm{i}\left(s_{\mathrm{f}}-s_{\mathrm{i}}\right) H} G_{b}^{(0)}\left(s_{\mathrm{i}}, 0\right)\right), & \text { if } s_{\mathrm{i}} \leqslant s_{\mathrm{f}}<t, \\ \operatorname{tr}_{b}\left(\rho_{b} G_{b}^{(0)}\left(2 t, s_{\mathrm{f}}\right) \mathrm{e}^{-\mathrm{i}\left(s_{\mathrm{i}}-s_{\mathrm{f}}\right) H} G_{b}^{(0)}\left(s_{\mathrm{i}}, 0\right)\right), & \text { if } t \leqslant s_{\mathrm{i}} \leqslant s_{\mathrm{f}}, \\ \operatorname{tr}_{b}\left(\rho_{b} G_{b}^{(0)}\left(2 t, s_{\mathrm{f}}\right) \mathrm{e}^{\mathrm{i}\left(s_{\mathrm{f}}-t\right) H} O \mathrm{e}^{-\mathrm{i}\left(t-s_{\mathrm{i}}\right) H} G_{b}^{(0)}\left(s_{\mathrm{i}}, 0\right)\right), & \text { if } s_{\mathrm{i}}<t \leqslant s_{\mathrm{f}} .\end{cases}
$$


Here the trace is taken only on the space of the bath $\mathcal{H}_{b}$, and hence $G\left(s_{\mathrm{f}}, s_{\mathrm{i}}\right)$ is an operator on $\mathcal{H}_{s}$. Following the same method from (3) to (7), we get the following Dyson series expansion for $G\left(s_{\mathrm{f}}, s_{\mathrm{i}}\right)$ :

$$
\begin{array}{r}
G\left(s_{\mathrm{f}}, s_{\mathrm{i}}\right)=\sum_{m=0}^{+\infty} \int_{s_{\mathrm{f}}>s_{m}>\cdots>s_{1}>s_{\mathrm{i}}}(-1)^{\#\{\boldsymbol{s}<t\}} \mathrm{i}^{m} \operatorname{tr}_{b}\left(\rho_{b} G_{b}^{(0)}\left(2 t, s_{\mathrm{f}}\right) G^{(0)}\left(s_{\mathrm{f}}, s_{m}\right) W G^{(0)}\left(s_{m}, s_{m-1}\right) W\right. \\
\left.\cdots W G^{(0)}\left(s_{2}, s_{1}\right) W G^{(0)}\left(s_{1}, s_{\mathrm{i}}\right) G_{b}^{(0)}\left(s_{\mathrm{i}}, 0\right)\right) \mathrm{d} s_{1} \cdots \mathrm{d} s_{m} .
\end{array}
$$

To apply the inchworm algorithm, we need the following two hypotheses, which are abstracted from the spin-Boson model studied in [4]:

(H1) The initial density matrix for the bath $\rho_{b}$ commutes with the Hamiltonian $H_{b}$. Physically, this condition holds when the bath is initially at the thermal equilibrium associated with the Hamiltonian $H_{b}$.

(H2) There exists a function $B(\cdot, \cdot)$ such that the following Wick's theorem holds:

$$
\mathcal{L}_{b}\left(s_{m}, \cdots, s_{1}\right)= \begin{cases}0, & \text { if } m \text { is odd } \\ \sum_{\mathfrak{q} \in \mathcal{Q}\left(s_{m}, \cdots, s_{1}\right)} \mathcal{L}(\mathfrak{q}), & \text { if } m \text { is even, }\end{cases}
$$

where the right hand side is given by all possible ordered pairings of the time points:

$$
\begin{aligned}
\mathcal{L}(\mathfrak{q})= & \prod_{\left(\tau_{1}, \tau_{2}\right) \in \mathfrak{q}} B\left(\tau_{1}, \tau_{2}\right), \\
\mathcal{Q}\left(s_{m}, \cdots, s_{1}\right)=\left\{\left\{\left(s_{j_{1}}, s_{k_{1}}\right), \cdots,\left(s_{j_{m / 2}}, s_{k_{m / 2}}\right)\right\} \mid\right. & \left\{j_{1}, \cdots, j_{m / 2}, k_{1}, \cdots, k_{m / 2}\right\}=\{1, \cdots, m\}, \\
& \left.s_{j_{l}} \leqslant s_{k_{l}} \text { for any } l=1, \cdots, m / 2\right\},
\end{aligned}
$$

When $m=0$, the value of $\mathcal{L}(\emptyset)$ is defined as 1 .

In hypothesis $(\mathrm{H} 2), \mathcal{Q}\left(s_{m}, \cdots, s_{1}\right)$ is the set of all possible ordered pairings of $\left\{s_{m}, \cdots, s_{1}\right\}$. For example,

$$
\begin{aligned}
& \mathcal{Q}\left(s_{2}, s_{1}\right)=\left\{\left\{\left(s_{1}, s_{2}\right)\right\}\right\}, \\
& \mathcal{Q}\left(s_{4}, s_{3}, s_{2}, s_{1}\right)=\left\{\left\{\left(s_{1}, s_{2}\right),\left(s_{3}, s_{4}\right)\right\},\left\{\left(s_{1}, s_{3}\right),\left(s_{2}, s_{4}\right)\right\},\left\{\left(s_{1}, s_{4}\right),\left(s_{2}, s_{3}\right)\right\}\right\} .
\end{aligned}
$$

We can also represent these sets by diagrams:

$$
\begin{aligned}
& \mathcal{Q}\left(s_{2}, s_{1}\right)=\left\{\frac{\overbrace{s_{1}}}{s_{1}}\right\},
\end{aligned}
$$

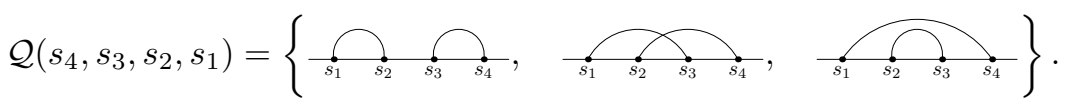

Manifestly, each arc stands for a pair formed by the labels on the two end points, and each diagram denotes a set of pairs.

As in the quantum field theory, Wick's theorem (hypothesis (H2)) turns integrals into diagrams, which allows us to use diagrammatic quantum Monte Carlo methods in the simulation. The first hypothesis (H1) associates the full propagators with observables. Precisely, when $s_{\mathrm{i}}<t<s_{\mathrm{f}}$ and $s_{\mathrm{i}}+s_{\mathrm{f}}=2 t$, we have

$$
G_{b}^{(0)}\left(s_{\mathrm{i}}, 0\right) \rho_{b} G_{b}^{(0)}\left(2 t, s_{\mathrm{f}}\right)=\mathrm{e}^{\mathrm{i}\left(2 t-s_{\mathrm{f}}\right) H_{b}} \rho_{b} \mathrm{e}^{-\mathrm{i} s_{\mathrm{i}} H_{b}}=\rho_{b} \mathrm{e}^{\mathrm{i}\left(2 t-s_{\mathrm{f}}-s_{\mathrm{i}}\right) H_{b}}=\rho_{b} .
$$

Thus by the cyclic property of the operator trace, we can derive from (13) that

$$
\operatorname{tr}_{s}\left(\rho_{s} G\left(s_{\mathrm{f}}, s_{\mathrm{i}}\right)\right)=\operatorname{tr}\left(\rho(0) \mathrm{e}^{\mathrm{i}\left(s_{\mathrm{f}}-t\right) H} O \mathrm{e}^{-\mathrm{i}\left(t-s_{\mathrm{i}}\right) H}\right)=\left\langle O\left(t-s_{\mathrm{i}}\right)\right\rangle
$$

which shows that the evolution of the observable from time 0 to $t$ can be fully obtained once the propagator $G\left(s_{\mathrm{f}}, s_{\mathrm{i}}\right)$ is solved for every pair of $s_{\mathrm{f}}$ and $s_{\mathrm{i}}$. In fact, when working with Wick's theorem (H2), we usually 
assume that $\rho_{b}=\exp \left(-\beta H_{b}\right)$, and thus the hypothesis (H1) is naturally fulfilled. By splitting system and bath parts and applying Wick's theorem (15), we get from (14) that

$$
G\left(s_{\mathrm{f}}, s_{\mathrm{i}}\right)=\sum_{\substack{m=0 \\ m \text { is even }}}^{+\infty} \int_{s_{\mathrm{f}}>s_{m}>\cdots>s_{1}>s_{\mathrm{i}}} \sum_{\mathfrak{q} \in \mathcal{Q}(\boldsymbol{s})}(-1)^{\#\{\boldsymbol{s}<t\}_{\mathrm{i}}{ }^{m} \mathcal{U}^{(0)}}\left(s_{\mathrm{f}}, \boldsymbol{s}, s_{\mathrm{i}}\right) \mathcal{L}(\mathfrak{q}) \mathrm{d} s_{1} \cdots \mathrm{d} s_{m} .
$$

Here the integral of $(-1)^{\#\{s<t\}_{i}{ }^{m} \mathcal{U}^{(0)}}\left(s_{\mathfrak{f}}, \boldsymbol{s}, s_{\mathrm{i}}\right) \mathcal{L}(\mathfrak{q})$ can also be represented by a diagram like Figure 3, which is interpreted by

- Each line segment connecting two adjacent time points labeled by $t_{\mathrm{s}}$ and $t_{\mathrm{f}}$ means a propagator $G_{s}^{(0)}\left(t_{\mathrm{f}}, t_{\mathrm{i}}\right)$.

- Each black dot introduces a perturbation operator $\pm \mathrm{i} W_{s}$, and we take the minus sign on the forward branch, and the plus sign on the backward branch. Here the label for time $t$, which separates the two branches of the Keldysh contour, is omitted. Additionally, each black dot also represents the integral with respect to the label over the interval from $s_{\mathrm{i}}$ to its next label.

- The arc connecting two time points $t_{\mathrm{s}}$ and $t_{\mathrm{f}}$ stands for $B\left(t_{\mathrm{s}}, t_{\mathrm{f}}\right)$.

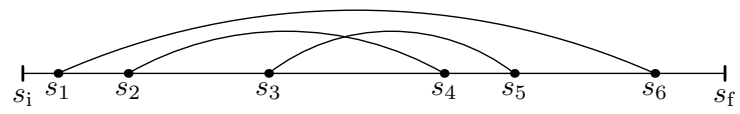

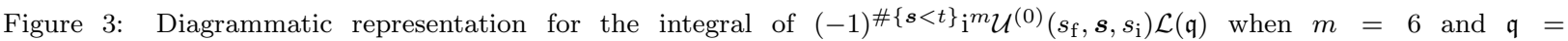
$\left\{\left(s_{1}, s_{6}\right),\left(s_{2}, s_{4}\right),\left(s_{3}, s_{5}\right)\right\}$.

Note that the branches are not explicitly labeled in Figure 3. The two end points $s_{\mathrm{i}}$ and $s_{\mathrm{f}}$ may both locate on the forward branch or the backward branch; they may also belong to different branches. Such a diagrammatic representation allows us to rewrite (19) as

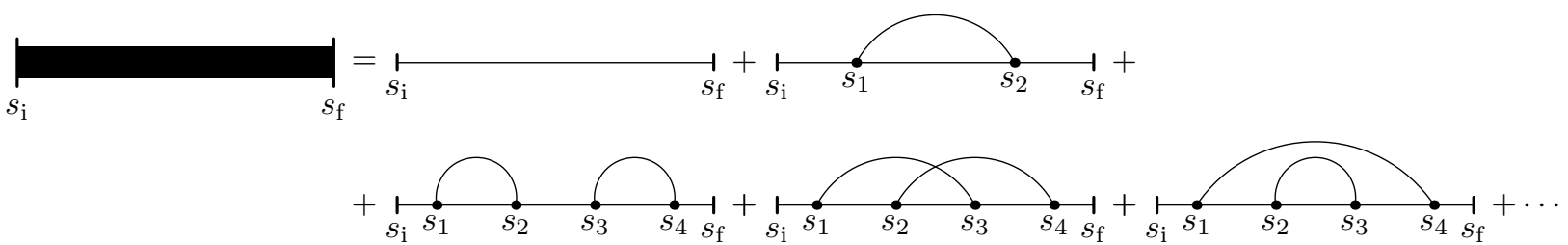

where the bold line on the left-hand side represents the full propagator $G\left(s_{\mathrm{f}}, s_{\mathrm{i}}\right)$. The Monte Carlo method based on (20) is referred to as "bare diagrammatic quantum Monte Carlo" method in [4], which is essentially identical to the Monte Carlo method based on the Dyson series expansion (3), except that the bath function $\mathcal{L}_{b}(\boldsymbol{s})$ is also evaluated by the Monte Carlo method.

Remark 1. The hypothesis (H1) aims to establish the relation between the full propagators and the observables. It can possibly be generalized for quantum Monte Carlo algorithms in other circumstances. For example, in the Anderson impurity model considered in [1], the Hamiltonian $H(t)$ depends on time and the initial state is the entire thermal equilibrium $\rho(0)=\exp (-\beta H(0))$, where the Keldysh contour needs to be extended by adding the imaginary Matsubara branch to get (18). Another example is [50], where the initial state includes chemical potentials, which does not exactly satisfy (H1) but a relation similar to (18) still holds.

Remark 2. The hypothesis (H2) is a bosonic Wick's theorem in physical terms. For fermions, a power of -1 needs to be added into the definition of $\mathcal{L}(\mathfrak{q})(16)$. While for definitness of presentation, we will stick to the bosonic representation in this work, the methodology and analysis in this paper are generalizable to the fermionic case as well. In practice, the algorithms based on bold diagrams have been successfully applied to the fermionic case such as the Anderson impurity model [1, 22]. 


\subsection{Description of the inchworm algorithm}

The inchworm algorithm uses another series expansion of $G\left(s_{\mathrm{f}}, s_{\mathrm{i}}\right)$ that leads to efficient use of results of previous time steps for future computations on the contour. Before introducing the inchworm series, we need the following definitions:

Definition 1 (Linked pairs and linked set of pairs). Two pairs of real numbers $\left(s_{1}, s_{2}\right)$ and $\left(\tau_{1}, \tau_{2}\right)$ satisfying $s_{1} \leqslant s_{2}$ and $\tau_{1} \leqslant \tau_{2}$ are linked if either of the following two statements holds:

1. $s_{1} \leqslant \tau_{1} \leqslant s_{2}$ and $\tau_{1} \leqslant s_{2} \leqslant \tau_{2}$.

2. $\tau_{1} \leqslant s_{1} \leqslant \tau_{2}$ and $s_{1} \leqslant \tau_{2} \leqslant s_{2}$.

For two sets of pairs $\mathfrak{q}_{1}$ and $\mathfrak{q}_{2}$, we say the two sets $\mathfrak{q}_{1}$ and $\mathfrak{q}_{2}$ are linked if there exists $\left(s_{1}, s_{2}\right) \in \mathfrak{q}_{1}$ and $\left(\tau_{1}, \tau_{2}\right) \in \mathfrak{q}_{2}$ such that $\left(s_{1}, s_{2}\right)$ and $\left(\tau_{1}, \tau_{2}\right)$ are linked.

Given a set of pairs $\mathfrak{q}$, we say $\mathfrak{q}$ is a linked set of pairs if it cannot be decomposed into the union of two sets of pairs that are not linked. We define

$$
\mathcal{Q}_{c}\left(s_{m}, \cdots, s_{1}\right)=\left\{\mathfrak{q} \in \mathcal{Q}\left(s_{m}, \cdots, s_{1}\right) \mid \mathfrak{q} \text { is linked }\right\} .
$$

For example, according to the above definition, when $s_{1}<s_{2}<s_{3}<s_{4}$, the pairs $\left(s_{1}, s_{3}\right)$ and $\left(s_{2}, s_{4}\right)$ are linked, while $\left(s_{1}, s_{4}\right)$ and $\left(s_{2}, s_{3}\right)$ are not, which can be also clearly seen from the diagrams:

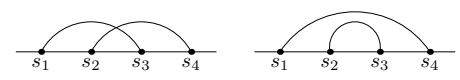

It is also clear that the set of pairs in the left diagram is a linked set, while the other one is not.

Definition 2 (Linked component decomposition). For any $\mathfrak{q} \in \mathcal{Q}\left(s_{m}, \cdots, s_{1}\right)$, there exists a collection of its disjoint subsets $\mathfrak{q}_{1}, \mathfrak{q}_{2}, \cdots, \mathfrak{q}_{n}$ such that

1. $\mathfrak{q}_{1}, \mathfrak{q}_{2}, \cdots, \mathfrak{q}_{n}$ are all linked sets of pairs;

2. $\mathfrak{q}=\mathfrak{q}_{1} \cup \mathfrak{q}_{2} \cup \cdots \cup \mathfrak{q}_{n}$;

3. $\mathfrak{q}_{n_{1}}$ and $\mathfrak{q}_{n_{2}}$ are not linked if $n_{1} \neq n_{2}$.

We call $\mathfrak{q}=\mathfrak{q}_{1} \cup \mathfrak{q}_{2} \cup \cdots \cup \mathfrak{q}_{n}$ the linked component decomposition of $\mathfrak{q}$.

An simple example of the linked component decomposition is given below by diagrammatic representations:

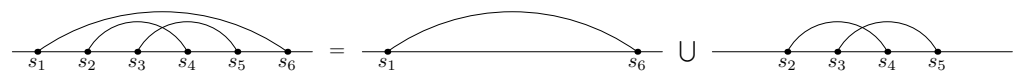

Definition 3 (Inchworm properness). Given a decreasing sequence of real numbers $s_{m}, \cdots, s_{1}$ and a real number $s_{\uparrow}$, a set of integer pairs $\mathfrak{q} \in \mathcal{Q}\left(s_{m}, \cdots, s_{1}\right)$ is called inchworm proper, if in its linked component decomposition $\mathfrak{q}=\mathfrak{q}_{1} \cup \mathfrak{q}_{2} \cup \cdots \cup \mathfrak{q}_{n}$, each $\mathfrak{q}_{k}$ contains at least one point greater than or equal to $s_{\uparrow}$. Below we use $\mathcal{Q}_{s_{\uparrow}}\left(s_{m}, \cdots, s_{1}\right)$ to denote the collection of all inchworm proper pair sets.

The structure of the set $\mathcal{Q}_{s_{\uparrow}}\left(s_{m}, \cdots, s_{1}\right)$ is determined by the relative position of $s_{\uparrow}$ in the decreasing sequence $s_{m}, \cdots, s_{1}$. For example, if $m=4$ and $s_{\uparrow} \in\left(s_{2}, s_{3}\right]$, then

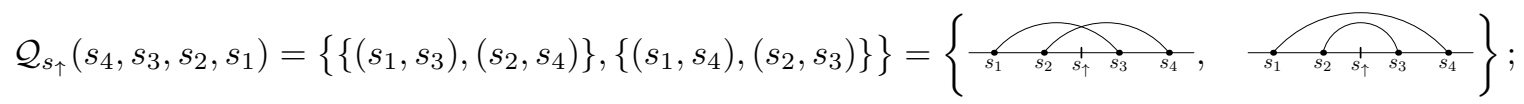

if $s_{\uparrow} \in\left(s_{3}, s_{4}\right]$, then $\mathcal{Q}_{s_{\uparrow}}\left(s_{4}, s_{3}, s_{2}, s_{1}\right)$ contains only one element:

$$
\mathcal{Q}_{s_{\uparrow}}\left(s_{4}, s_{3}, s_{2}, s_{1}\right)=\left\{\left\{\left(s_{1}, s_{3}\right),\left(s_{2}, s_{4}\right)\right\}\right\}=\left\{\begin{array}{llll}
\overbrace{s_{1}} & \overbrace{s_{2}} & s_{s_{\uparrow}} & s_{4}
\end{array}\right\} .
$$

In fact, if $s_{\uparrow} \in\left(s_{m-1}, s_{m}\right]$, the number of sets of pairs in the linked component decomposition of an inchworm proper set $\mathfrak{q}$ must be 1 , which means $\mathfrak{q}$ is linked. Therefore we have

$$
\mathcal{Q}_{s_{\uparrow}}\left(s_{m}, \cdots, s_{1}\right)=\mathcal{Q}_{c}\left(s_{m}, \cdots, s_{1}\right), \quad \text { if } s_{m-1}<s_{\uparrow} \leqslant s_{m} .
$$

Based on the definition of inchworm proper set of pairs, we have the following theorem: 
Theorem 1. Suppose the Dyson series (19) is absolutely convergent in the sense that

$$
\sum_{\substack{m=0 \\ m \text { is even }}}^{+\infty} \int_{s_{\mathrm{f}}>s_{m}>\cdots>s_{1}>s_{\mathrm{i}}}\left\|\mathcal{U}\left(s_{\mathrm{f}}, \boldsymbol{s}, s_{\mathrm{i}}\right)\right\|_{s}\left|\sum_{\mathfrak{q} \in \mathcal{Q}(\boldsymbol{s})} \mathcal{L}(\mathfrak{q})\right| \mathrm{d} s_{1} \cdots \mathrm{d} s_{m}<+\infty, \quad \forall s_{\mathrm{i}}, s_{\mathrm{f}} \in[0,2 t],
$$

where $\|\cdot\|_{s}$ is the operator norm in the Hilbert space $\mathcal{H}_{s}$. For any $s_{\uparrow} \in\left(s_{\mathrm{i}}, s_{\mathrm{f}}\right)$, we have

$$
\begin{aligned}
& G\left(s_{\mathrm{f}}, s_{\mathrm{i}}\right)=\mathcal{G}_{s_{\uparrow}}\left(s_{\mathrm{f}}, s_{\mathrm{i}}\right)+\sum_{\substack{m=2 \\
m \text { is even }}}^{+\infty} \int_{s_{\mathrm{f}}>s_{m}>\cdots>s_{1}>s_{\mathrm{i}}}(-1)^{\#\{\boldsymbol{s}<t\}_{\mathrm{i}} m}\left(\sum_{\mathfrak{q} \in \mathcal{Q}_{s_{\uparrow}}(\boldsymbol{s})} \mathcal{L}(\mathfrak{q})\right) \times \\
& \mathcal{G}_{s_{\uparrow}}\left(s_{\mathrm{f}}, s_{m}\right) W_{s} \mathcal{G}_{s_{\uparrow}}\left(s_{m}, s_{m-1}\right) W_{s} \cdots W_{s} \mathcal{G}_{s_{\uparrow}}\left(s_{2}, s_{1}\right) W_{s} \mathcal{G}_{s_{\uparrow}}\left(s_{1}, s_{\mathrm{i}}\right) \mathrm{d} s_{1} \cdots \mathrm{d} s_{m},
\end{aligned}
$$

where

$$
\mathcal{G}_{s_{\uparrow}}\left(s_{\mathrm{f}}, s_{\mathrm{i}}\right)= \begin{cases}G\left(s_{\mathrm{f}}, s_{\mathrm{i}}\right), & \text { if } s_{\mathrm{i}} \leqslant s_{\mathrm{f}} \leqslant s_{\uparrow}, \\ G_{s}^{(0)}\left(s_{\mathrm{f}}, s_{\mathrm{i}}\right), & \text { if } s_{\uparrow}<s_{\mathrm{i}} \leqslant s_{\mathrm{f}}, \\ G_{s}^{(0)}\left(s_{\mathrm{f}}, s_{\uparrow}\right) G\left(s_{\uparrow}, s_{\mathrm{i}}\right), & \text { if } s_{\mathrm{i}} \leqslant s_{\uparrow}<s_{\mathrm{f}} .\end{cases}
$$

Note that the right-hand side of (23) has a very similar structure to (19), except that the bare Green's function $G_{s}^{(0)}$ is replaced by $\mathcal{G}_{s \uparrow}$ and the sum consists only of "inchworm proper diagrams", which will be further discussed below. This theorem shows that $G\left(s_{\mathrm{f}}, s_{\mathrm{i}}\right)$ can be evaluated by the Monte Carlo simulation of the right-hand side of (23) based on the knowledge of $G\left(\tau_{2}, \tau_{1}\right)$ for all $s_{\mathrm{i}}<\tau_{1}<\tau_{2}<s_{\uparrow}$. Therefore the algorithm can be designed as follows:

Choose a time step $\Delta t=t / N$

for $s_{\mathrm{f}}$ from 0 to $2 t$ with step $\Delta t$

for $s_{\mathrm{i}}$ from $s_{\mathrm{f}}$ to 0 with step $-\Delta t$

Use (23) to evaluate $G\left(s_{\mathrm{f}}, s_{\mathrm{i}}\right)$ by the Monte Carlo method

end for

end for

This algorithm computes all $G\left(s_{\mathrm{f}}, s_{\mathrm{i}}\right)$ when $s_{\mathrm{i}}$ and $s_{\mathrm{f}}$ are multiples of $\Delta t$. When evaluating the right-hand side of (23), interpolation might be needed to get $G\left(\tau_{2}, \tau_{1}\right)$ when $\tau_{1}$ or $\tau_{2}$ is not an integer multiple of $\Delta t$. Details about the interpolation will be given in Section 6 .

A rigorous proof of Theorem 1 will be given in the next section. Here we would like to provide the diagrammatic understanding of this algorithm, following [4, 5]. As already mentioned, on the right-hand side of (23), only "inchworm proper diagrams" appear, for example,

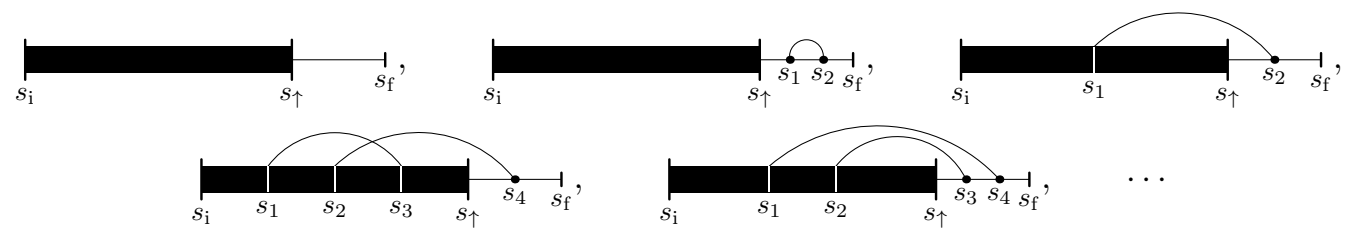

These diagrams are interpreted as follows:

- Each bold line segment connecting two adjacent time points labeled by $t_{\mathrm{i}}$ and $t_{\mathrm{f}}$ means a full propagator $G\left(t_{\mathrm{f}}, t_{\mathrm{i}}\right)$.

- Each thin line segment connecting two adjacent time points labeled by $t_{\mathrm{i}}$ and $t_{\mathrm{f}}$ means a propagator $G_{s}^{(0)}\left(t_{\mathrm{f}}, t_{\mathrm{i}}\right)$.

- Each black dot introduces a perturbation operator $\pm \mathrm{i} W_{s}$, which takes the minus sign on the forward branch and the plus sign on the backward branch. It also introduces an integral with respect to the label from $s_{\uparrow}$ to the next label. 
- Each white vertical line introduces a perturbation operator $\pm \mathrm{i} W_{s}$, which takes the minus sign on the forward branch and the plus sign on the backward branch. It also introduces an integral with respect to the label from $s_{\mathrm{i}}$ to the next label.

- The arc connecting two time points $t_{\mathrm{i}}$ and $t_{\mathrm{f}}$ stands for $B\left(t_{\mathrm{i}}, t_{\mathrm{f}}\right)$.

By (20), we can see that each bold line in the above diagrams is a sum consisting of infinite "thin diagrams", which means that (20) is a partial resummation of the Dyson series expansion. Therefore it can be expected that (23) converges faster than (19). It is also worth mentioning that the following diagrams are not included in the inchworm series (23):
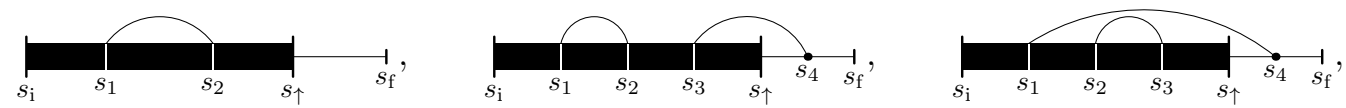

since the terms presented by these diagrams have actually appeared in other diagrams, which are respectively
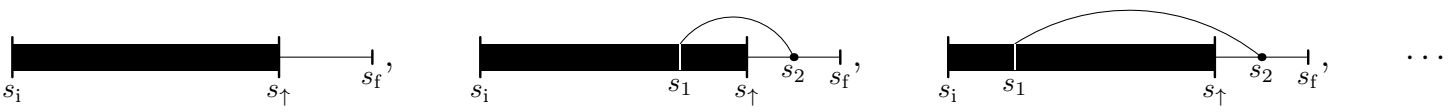

Below we use a diagrammatic equation to summarize the idea of the inchworm algorithm:

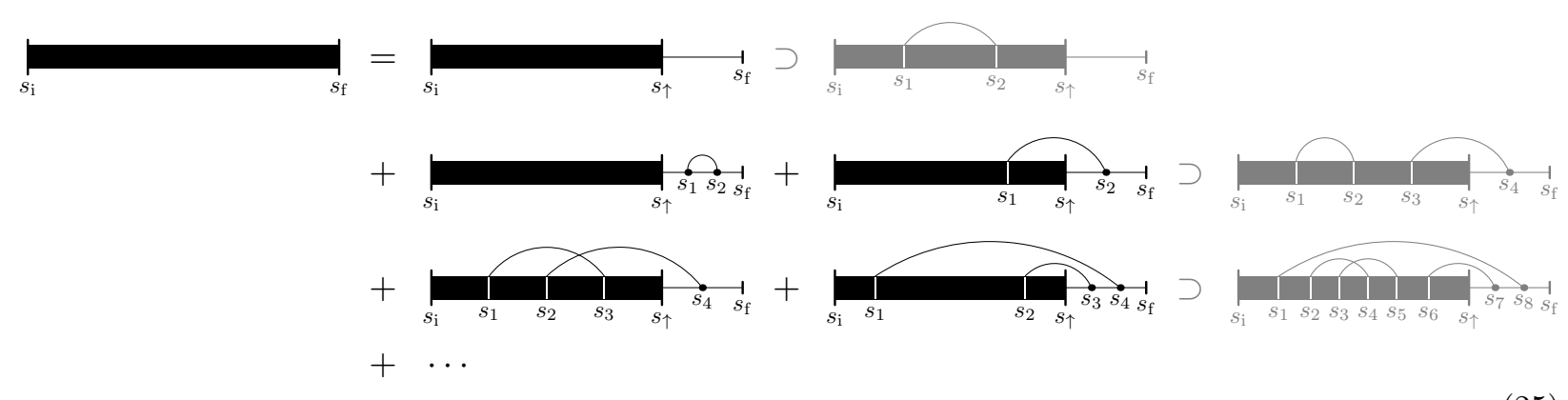

The proof of Theorem 1 follows such understanding of the inchworm series. However, to make sure no diagrams are missed or double-counted in this equation, we need to interpret these diagrammatic equations precisely using mathematical equations, which will be detailed in the following section.

\subsection{Proof of Theorem [1}

This section is devoted to the proof of (23), which is to equate the right-hand side of (23) and the series (19). To see this, we will first introduce $s_{\uparrow}$ to the expansion of $G\left(s_{\mathrm{f}}, s_{\mathrm{i}}\right)$ by the following lemma:

Lemma 1. When the Dyson series (19) is absolutely convergent in the sense of (22), for any $s_{\uparrow} \in\left(s_{\mathrm{i}}, s_{\mathrm{f}}\right)$, it holds that

$$
\begin{aligned}
& G\left(s_{\mathrm{f}}, s_{\mathrm{i}}\right)=G_{s}^{(0)}\left(s_{\mathrm{f}}, s_{\uparrow}\right) G\left(s_{\uparrow}, s_{\mathrm{i}}\right)+ \sum_{\substack{m=2 \\
m \text { is even }}}^{+\infty} \sum_{p=0}^{m-1} \int_{s_{\mathrm{f}}>s_{m}>\cdots>s_{p+1}>s_{\uparrow}} \int_{s_{\uparrow}>s_{p}>\cdots>s_{1}>s_{\mathrm{i}}} \sum_{\mathfrak{q} \in \mathcal{Q}(\boldsymbol{s})} \\
&(-1)^{\#\{\boldsymbol{s}<t\}} \mathrm{i}^{m} \mathcal{U}^{(0)}\left(s_{\mathrm{f}}, \boldsymbol{s}, s_{\mathrm{i}}\right) \mathcal{L}(\mathfrak{q}) \mathrm{d} s_{1} \cdots \mathrm{d} s_{p} \mathrm{~d} s_{p+1} \cdots \mathrm{d} s_{m} .
\end{aligned}
$$

Proof. For any positive integer $m$, it holds that

$$
\int_{s_{\mathrm{f}}>s_{m}>\cdots>s_{1}>s_{\mathrm{i}}} \varphi(\boldsymbol{s}) \mathrm{d} s_{1} \cdots \mathrm{d} s_{m}=\sum_{p=0}^{m} \int_{s_{\mathrm{f}}>s_{m}>\cdots>s_{p+1}>s_{\uparrow}} \int_{s_{\uparrow}>s_{p}>\cdots>s_{1}>s_{\mathrm{i}}} \varphi(\boldsymbol{s}) \mathrm{d} s_{1} \cdots \mathrm{d} s_{p} \mathrm{~d} s_{p+1} \cdots \mathrm{d} s_{m}
$$


for any function $\varphi$. This can be proven by mathematical induction since when the above equation holds for some $m$, we have

$$
\begin{aligned}
& \int_{s_{\mathrm{f}}>s_{m+1}>\cdots>s_{1}>s_{\mathrm{i}}} \varphi(\boldsymbol{s}) \mathrm{d} s_{1} \cdots \mathrm{d} s_{m+1}=\int_{s_{\mathrm{i}}}^{s_{\mathrm{f}}}\left(\int_{s_{m+1}>s_{m}>\cdots>s_{1}>s_{\mathrm{i}}} \varphi(\boldsymbol{s}) \mathrm{d} s_{1} \cdots \mathrm{d} s_{m}\right) \mathrm{d} s_{m+1} \\
= & \int_{s_{\mathrm{i}}}^{s_{\uparrow}}\left(\int_{s_{m+1}>s_{m}>\cdots>s_{1}>s_{\mathrm{i}}} \varphi(\boldsymbol{s}) \mathrm{d} s_{1} \cdots \mathrm{d} s_{m}\right) \mathrm{d} s_{m+1}+\int_{s_{\uparrow}}^{s_{\mathrm{f}}}\left(\int_{s_{m+1}>s_{m}>\cdots>s_{1}>s_{\mathrm{i}}} \varphi(\boldsymbol{s}) \mathrm{d} s_{1} \cdots \mathrm{d} s_{m}\right) \mathrm{d} s_{m+1} \\
= & \int_{s_{\uparrow}>s_{m+1}>\cdots>s_{1}>s_{\mathrm{i}}} \varphi(\boldsymbol{s}) \mathrm{d} s_{1} \cdots \mathrm{d} s_{m+1} \\
& +\int_{s_{\uparrow}}^{s_{\mathrm{f}}}\left(\sum_{p=0}^{m} \int_{s_{m+1}>s_{m}>\cdots>s_{p+1}>s_{\uparrow}} \int_{s_{\uparrow}>s_{p}>\cdots>s_{1}>s_{\mathrm{i}}} \varphi(\boldsymbol{s}) \mathrm{d} s_{1} \cdots \mathrm{d} s_{p} \mathrm{~d} s_{p+1} \cdots \mathrm{d} s_{m}\right) \mathrm{d} s_{m+1} \\
= & \int_{s_{\uparrow}>s_{m+1}>\cdots>s_{1}>s_{\mathrm{i}}}^{m} \varphi(\boldsymbol{s}) \mathrm{d} s_{1} \cdots \mathrm{d} s_{m+1} \\
& +\sum_{p=0}^{m} \int_{s_{\mathrm{f}}>s_{m+1}>\cdots>s_{p+1}>s_{\uparrow}} \int_{s_{\uparrow}>s_{p}>\cdots>s_{1}>s_{\mathrm{i}}} \varphi(s) \mathrm{d} s_{1} \cdots \mathrm{d} s_{p} \mathrm{~d} s_{p+1} \cdots \mathrm{d} s_{m+1} \\
= & \sum_{p=0}^{m+1} \int_{s_{\mathrm{f}}>s_{m+1}>\cdots>s_{p+1}>s_{\uparrow}} \int_{s_{\uparrow}>s_{p}>\cdots>s_{1}>s_{\mathrm{i}}} \varphi(\boldsymbol{s}) \mathrm{d} s_{1} \cdots \mathrm{d} s_{p} \mathrm{~d} s_{p+1} \cdots \mathrm{d} s_{m+1},
\end{aligned}
$$

and it is obvious that (27) holds for $m=1$. By (27), we can rewrite (19) as

$$
\begin{aligned}
& G\left(s_{\mathrm{f}}, s_{\mathrm{i}}\right)=\sum_{\substack{m=0 \\
m \text { is even }}}^{+\infty} \sum_{p=0}^{m} \int_{s_{\mathrm{f}}>s_{m}>\cdots>s_{p+1}>s_{\uparrow}} \int_{s_{\uparrow}>s_{p}>\cdots>s_{1}>s_{\mathrm{i}}} \sum_{\mathfrak{q} \in \mathcal{Q}(s)} \\
& (-1)^{\#\{\boldsymbol{s}<t\}_{\mathrm{i}}{ }^{m} \mathcal{U}^{(0)}}\left(s_{\mathrm{f}}, \boldsymbol{s}, s_{\mathrm{i}}\right) \mathcal{L}(\mathfrak{q}) \mathrm{d} s_{1} \cdots \mathrm{d} s_{p} \mathrm{~d} s_{p+1} \cdots \mathrm{d} s_{m} \\
& =\sum_{\substack{m=0 \\
m \text { is even }}}^{+\infty} \int_{s_{\uparrow}>s_{m}>\cdots>s_{1}>s_{\mathrm{i}}} \sum_{\mathfrak{q} \in \mathcal{Q}(\boldsymbol{s})}(-1)^{\#\{\boldsymbol{s}<t\}_{\mathrm{i}} m} \mathcal{U}^{(0)}\left(s_{\mathrm{f}}, \boldsymbol{s}, s_{\mathrm{i}}\right) \mathcal{L}(\mathfrak{q}) \mathrm{d} s_{1} \cdots \mathrm{d} s_{m} \\
& +\sum_{\substack{m=2 \\
m \text { is even }}}^{+\infty} \sum_{p=0}^{m-1} \int_{s_{\mathrm{f}}>s_{m}>\cdots>s_{p+1}>s_{\uparrow}} \int_{s_{\uparrow}>s_{p}>\cdots>s_{1}>s_{\mathrm{i}}} \sum_{\mathfrak{q} \in \mathcal{Q}(\boldsymbol{s})}
\end{aligned}
$$

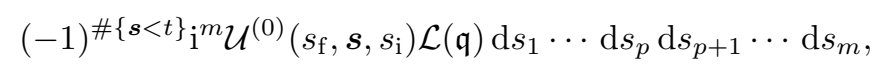

where we have used the absolute convergence (22) to ensure the validity of the second equality. When $s_{m}<s_{\uparrow}$, we have $\mathcal{U}^{(0)}\left(s_{\mathrm{f}}, \boldsymbol{s}, s_{\mathrm{i}}\right)=G_{s}^{(0)}\left(s_{\mathrm{f}}, s_{\uparrow}\right) \mathcal{U}^{(0)}\left(s_{\uparrow}, \boldsymbol{s}, s_{\mathrm{i}}\right)$. Hence

$$
\begin{aligned}
& \sum_{\substack{m=0 \\
m \text { is even }}}^{+\infty} \int_{s_{\uparrow}>s_{m}>\cdots>s_{1}>s_{\mathrm{i}}} \sum_{\mathfrak{q} \in \mathcal{Q}(\boldsymbol{s})}(-1)^{\#\{\boldsymbol{s}<t\}_{\mathrm{i}}^{m} \mathcal{U}^{(0)}\left(s_{\mathrm{f}}, \boldsymbol{s}, s_{\mathrm{i}}\right) \mathcal{L}(\mathfrak{q}) \mathrm{d} s_{1} \cdots \mathrm{d} s_{m}} \\
= & G_{s}^{(0)}\left(s_{\mathrm{f}}, s_{\uparrow}\right) \sum_{\substack{m=0 \\
m}}^{+\infty} \int_{s_{\uparrow}>s_{m}>\cdots>s_{1}>s_{\mathrm{i}}} \sum_{\mathfrak{q} \in \mathcal{Q}(\boldsymbol{s})}(-1)^{\#\{\boldsymbol{s}<t\}_{\mathrm{i}} m} \mathcal{U}^{(0)}\left(s_{\uparrow}, \boldsymbol{s}, s_{\mathrm{i}}\right) \mathcal{L}(\mathfrak{q}) \mathrm{d} s_{1} \cdots \mathrm{d} s_{m}=G_{s}^{(0)}\left(s_{\mathrm{f}}, s_{\uparrow}\right) G\left(s_{\uparrow}, s_{\mathrm{i}}\right) .
\end{aligned}
$$

Inserting this equation into (28) yields our conclusion (26).

By the above lemma, we have extracted the first diagram in (25) from the definition of the bold line (20). Our next step is to introduce the inchworm proper pairings to the series expansion. The basic idea 
is to decompose every set of pairs into the union of one inchworm proper subset and several other unlinked subsets. The result reads

Lemma 2. When the Dyson series (19) is absolutely convergent in the sense of (22), for any $s_{\uparrow} \in\left(s_{\mathrm{i}}, s_{\mathrm{f}}\right)$, it holds that

$$
\begin{aligned}
& G\left(s_{\mathrm{f}}, s_{\mathrm{i}}\right)=G_{s}^{(0)}\left(s_{\mathrm{f}}, s_{\uparrow}\right) G\left(s_{\uparrow}, s_{\mathrm{i}}\right)+\sum_{\substack{\tilde{m}=2 \\
\tilde{m} \text { is even }}}^{+\infty} \sum_{\tilde{p}=0}^{\tilde{m}-1} \int_{s_{\mathrm{f}}>\tilde{s}_{\tilde{m}}>\cdots>\tilde{s}_{\tilde{p}+1}>s_{\uparrow}} \int_{s_{\uparrow}>\tilde{s}_{\tilde{p}}>\cdots>\tilde{s}_{1}>s_{\mathrm{i}}} \\
& \sum_{\substack{p=0 \\
\tilde{p}-p \text { is even }}}^{\tilde{p}} \sum_{\substack{n_{p}=0 \\
n_{p} \text { is even }}}^{\tilde{p}-p} \sum_{\substack{n_{p-1}=0 \\
n_{p-1} \text { is even }}}^{\tilde{p}-p-n_{p}} \cdots \sum_{\begin{array}{c}
n_{1}=0 \\
n_{1} \text { is even }
\end{array}}^{\tilde{p}-p-n_{p}-\cdots-n_{2}} \sum_{\mathfrak{q} \in \mathcal{Q}_{s_{\uparrow}}(\boldsymbol{s})} \sum_{\mathfrak{q}_{p} \in \mathcal{Q}\left(\boldsymbol{s}^{(p)}\right)} \cdots \sum_{\mathfrak{q}_{0} \in \mathcal{Q}\left(\boldsymbol{s}^{(0)}\right)}
\end{aligned}
$$

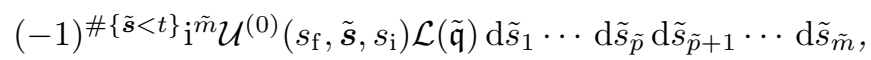

where the notations are

- $\tilde{\boldsymbol{s}}=\left(\tilde{s}_{\tilde{m}}, \cdots, \tilde{s}_{1}\right)$;

- $\tilde{\mathfrak{q}}=\mathfrak{q} \cup \mathfrak{q}_{p} \cup \cdots \cup \mathfrak{q}_{0} ;$

- $\boldsymbol{s}, \boldsymbol{s}^{(p)}, \cdots, \boldsymbol{s}^{(0)}$ are all subsequences of $\tilde{\boldsymbol{s}}$, defined by

$$
\tilde{\boldsymbol{s}}=\left(s_{m}, \cdots, s_{p+1}, \boldsymbol{s}^{(p)}, s_{p}, \boldsymbol{s}^{(p-1)}, s_{p-1}, \cdots, \boldsymbol{s}^{(1)}, s_{1}, \boldsymbol{s}^{(0)}\right),
$$

where $m=\tilde{m}+p-\tilde{p}$ and $s=\left(s_{m}, \cdots, s_{1}\right)$.

Proof. By comparing (29) with (26), we construct the following map for given $\tilde{m}, \tilde{p}$ and a decreasing sequence $\tilde{s}:$

$$
\begin{array}{ccc}
\mathcal{P} & \rightarrow & \mathcal{Q}(\tilde{\boldsymbol{s}}) \\
\left(p, n_{p}, \cdots, n_{1}, \mathfrak{q}, \mathfrak{q}_{p}, \cdots, \mathfrak{q}_{0}\right) & \mapsto & \mathfrak{q} \cup \mathfrak{q}_{p} \cup \cdots \cup \mathfrak{q}_{0}
\end{array}
$$

where

$$
\begin{aligned}
& \mathcal{P}=\left\{\left(p, n_{p}, \cdots, n_{1}, \mathfrak{q}, \mathfrak{q}_{p}, \cdots, \mathfrak{q}_{0}\right) \mid\right. p \in\{\tilde{p}, \tilde{p}-2, \cdots, \tilde{p}-2\lfloor\tilde{p} / 2\rfloor\} ; n_{1}+\cdots+n_{p} \leqslant \tilde{p}-p \\
&\left.n_{p}, \cdots, n_{1} \text { are even; } \mathfrak{q} \in \mathcal{Q}_{s_{\uparrow}}(\boldsymbol{s}) ; \mathfrak{q}_{p} \in \mathcal{Q}\left(\boldsymbol{s}^{(p)}\right) ; \cdots ; \mathfrak{q}_{0} \in \mathcal{Q}\left(\boldsymbol{s}^{(0)}\right)\right\} .
\end{aligned}
$$

The subsequences $\boldsymbol{s}, \boldsymbol{s}^{(p)}, \cdots, \boldsymbol{s}^{(0)}$ are defined as in the lemma. If (30) is a bijection, then (29) is a rearrangement of the series (26). The equality (29) then follows by the absolute convergence of the Dyson series.

Now it remains only to show that (30) is one-to-one. The definition of $\mathcal{P}$ shows that in a map $\left(p, n_{p}, \cdots, n_{1}, \mathfrak{q}, \mathfrak{q}_{p}, \cdots, \mathfrak{q}_{0}\right) \mapsto \tilde{\mathfrak{q}}$, if $\mathfrak{q}$ and $\tilde{\mathfrak{q}}$ are given, other parameters are automatically determined by

$$
\begin{aligned}
& p=2|\mathfrak{q}|-(\tilde{m}-\tilde{p}), \quad \mathfrak{q}_{0}=\left\{\left(\tau_{1}, \tau_{2}\right) \in \tilde{\mathfrak{q}} \mid \tau_{1}<\tau_{2}<s_{1}\right\}, \\
& \mathfrak{q}_{k}=\left\{\left(\tau_{1}, \tau_{2}\right) \in \tilde{\mathfrak{q}} \mid s_{k}<\tau_{1}<\tau_{2}<s_{k+1}\right\}, \quad k=1, \cdots, p, \\
& n_{k}=2\left|\mathfrak{q}_{k}\right|, \quad k=1, \cdots, p .
\end{aligned}
$$

Here $2|\mathfrak{q}|\left(2\left|\mathfrak{q}_{k}\right|\right)$ is actually the number of time points in $\mathfrak{q}\left(\mathfrak{q}_{k}\right)$. Meanwhile, the set of pairs $\mathfrak{q}$ satisfies

1. $\mathfrak{q}$ is not linked to any other pair in $\tilde{\mathfrak{q}}$;

2. $\mathfrak{q} \in \mathcal{Q}_{s_{\uparrow}}(\boldsymbol{s})$ and therefore contains $\tilde{s}_{\tilde{p}+1}, \cdots, \tilde{s}_{\tilde{m}}$.

Now we consider an arbitrary set of pairs $\tilde{\mathfrak{q}} \in \mathcal{Q}(\tilde{\boldsymbol{s}})$ with its linked component decomposition being $\tilde{\mathfrak{q}}=$ $\tilde{\mathfrak{q}}_{1} \cup \cdots \cup \tilde{\mathfrak{q}}_{n}$. The only subset of $\tilde{\mathfrak{q}}$ satisfying the above two conditions is

$$
\mathfrak{q}=\bigcup\left\{\tilde{\mathfrak{q}}_{k} \mid \tilde{\mathfrak{q}}_{k} \text { contains a pair }\left(\tau_{1}, \tau_{2}\right) \text { with } \tau_{2}>\tilde{s}_{\tilde{p}}\right\}
$$

Taking such a $\mathfrak{q}$ and applying (31) to find other parameters, we obtain an inverse image of $\tilde{\mathfrak{q}}$. This inverse image is unique due to the uniqueness of $\mathfrak{q}$, which yields that (30) is a bijection. 
Lemma 3. When the Dyson series (19) is absolutely convergent in the sense of (22), for any $s_{\uparrow} \in\left(s_{\mathrm{i}}, s_{\mathrm{f}}\right)$, it holds that

$$
\begin{aligned}
& G\left(s_{\mathrm{f}}, s_{\mathrm{i}}\right)=G^{(0)}\left(s_{\mathrm{f}}, s_{\uparrow}\right) G\left(s_{\uparrow}, s_{\mathrm{i}}\right)+\sum_{\substack{m=2 \\
m \text { is even }}}^{+\infty} \sum_{p=0}^{m-1} \sum_{\substack{n_{p}=0 \\
n_{p} \text { is even }}}^{+\infty} \sum_{\substack{n_{0}=0 \\
n_{0} \text { is even }}}^{+\infty} \int_{s_{\mathrm{f}}>\tilde{s}_{\tilde{m}}>\cdots>\tilde{s}_{\tilde{p}+1}>s_{\uparrow}} \int_{s_{\uparrow}>\tilde{s}_{\tilde{p}}>\cdots>\tilde{s}_{1}>s_{\mathrm{i}}}
\end{aligned}
$$

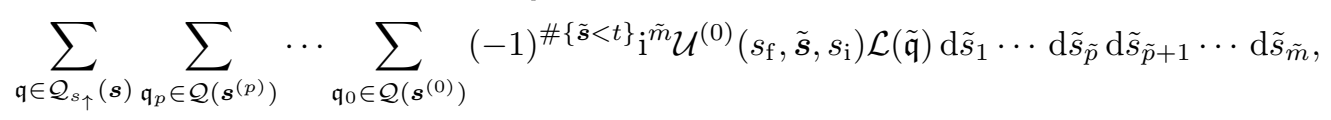

where $\tilde{m}=m+n_{0}+\cdots+n_{p}$, and other parameters are defined in the same way as in Lemma Q2.

Proof. It is not difficult to see that the map

$$
\begin{array}{ccc}
\mathcal{P} & \rightarrow & \widetilde{\mathcal{P}} \\
\left(m, p, n_{p}, \cdots, n_{0}\right) & \mapsto & \left(\tilde{m}, \tilde{p}, p, n_{p}, \cdots, n_{1}\right)
\end{array}
$$

with $\tilde{m}=m+n_{p}+\cdots+n_{0}$ and $\tilde{p}=p+n_{p}+\cdots+n_{0}$ is a bijection. Here

$$
\begin{aligned}
& \mathcal{P}=\left\{\left(m, p, n_{p}, \cdots, n_{0}\right) \mid m, n_{p}, \cdots, n_{0} \text { are positive and even; } p \in\{0, \cdots, m-1\}\right\}, \\
& \widetilde{\mathcal{P}}=\left\{\left(\tilde{m}, \tilde{p}, p, n_{p}, \cdots, n_{1}\right) \mid \tilde{m}, n_{p}, \cdots, n_{1}\right. \text { are positive and even; } \\
& \left.\tilde{p} \in\{0, \cdots, \tilde{m}-1\} ; p \in\{0,2, \cdots, 2\lfloor\tilde{p} / 2\rfloor\} ; p+n_{p}+\cdots+n_{1} \leqslant \tilde{p}\right\} .
\end{aligned}
$$

Hence (32) is a rearrangement of the series (29), and therefore (32) follows by the absolute convergence of the Dyson series.

Now we are ready to carry out the proof of the inchworm series (23):

Proof of Theorem 1. Following the notations in Lemma 2 and 3, we have the following identities:

$$
\begin{gathered}
\mathcal{U}^{(0)}(\boldsymbol{s})=\mathcal{U}^{(0)}\left(s_{\mathrm{f}}, s_{m}, \cdots, s_{p+1}, s_{\uparrow}\right) \mathcal{U}^{(0)}\left(s_{\uparrow}, \boldsymbol{s}^{(p)}, s_{p}\right) W_{s} \mathcal{U}^{(0)}\left(s_{p}, \boldsymbol{s}^{(p-1)}, s_{p-1}\right) W_{s} \cdots W_{s} \mathcal{U}^{(0)}\left(s_{1}, \boldsymbol{s}^{(0)}, s_{\mathrm{i}}\right) \\
\#\{\tilde{\boldsymbol{s}}<t\}=\#\{\boldsymbol{s}<t\}+\#\left\{\boldsymbol{s}^{(p)}<t\right\}+\cdots+\#\left\{\boldsymbol{s}^{(0)}<t\right\}, \quad \mathcal{L}(\tilde{\mathfrak{q}})=\mathcal{L}(\mathfrak{q}) \mathcal{L}\left(\mathfrak{q}_{p}\right) \cdots \mathcal{L}\left(\mathfrak{q}_{0}\right)
\end{gathered}
$$

Substituting these equalities to (32) yields

$$
\begin{aligned}
& G\left(s_{\mathrm{f}}, s_{\mathrm{i}}\right)=G_{s}^{(0)}\left(s_{\mathrm{f}}, s_{\uparrow}\right) G\left(s_{\uparrow}, s_{\mathrm{i}}\right)+\sum_{\substack{m=2 \\
m \text { is even }}}^{+\infty} \sum_{\substack{p=0 \\
n_{p} \text { is even }}}^{m-1} \sum_{\substack{n_{p}=0 \\
n_{p}}}^{+\infty} \sum_{\substack{n_{0}=0 \\
n_{0} \text { is even }}}^{+\infty} \\
& \int_{s_{\uparrow}}^{s_{\mathrm{f}}} \int_{s_{\uparrow}}^{s_{m}} \cdots \int_{s_{\uparrow}}^{s_{p+2}} \int_{s_{\mathrm{i}}}^{s_{\uparrow}} \int_{s_{\mathrm{i}}}^{s_{p}} \cdots \int_{s_{\mathrm{i}}}^{s_{2}} \int_{\boldsymbol{s}^{(p)} \subset\left(s_{p}, s_{\uparrow}\right)} \int_{\boldsymbol{s}^{(p-1)} \subset\left(s_{p-1}, s_{p}\right)} \cdots \int_{\boldsymbol{s}^{(1)} \subset\left(s_{1}, s_{2}\right)} \int_{\boldsymbol{s}^{(0)} \subset\left(s_{\mathrm{i}}, s_{1}\right)} \\
& \sum_{\mathfrak{q} \in \mathcal{Q}_{s_{\uparrow}}(\boldsymbol{s})} \sum_{\mathfrak{q}_{p} \in \mathcal{Q}\left(\boldsymbol{s}^{(p)}\right)} \cdots \sum_{\mathfrak{q}_{0} \in \mathcal{Q}\left(\boldsymbol{s}^{(0)}\right)}(-1)^{\#\{\boldsymbol{s}<t\}+\#\left\{\boldsymbol{s}^{(p)}<t\right\}+\cdots+\#\left\{\boldsymbol{s}^{(0)}<t\right\}} \mathrm{i}^{m+n_{p}+\cdots+n_{0}} \times \\
& \mathcal{U}^{(0)}\left(s_{\mathrm{f}}, s_{m}, \cdots, s_{p+1}, s_{\uparrow}\right) \mathcal{U}^{(0)}\left(s_{\uparrow}, \boldsymbol{s}^{(p)}, s_{p}\right) W_{s} \mathcal{U}^{(0)}\left(s_{p}, \boldsymbol{s}^{(p-1)}, s_{p-1}\right) W_{s} \cdots W_{s} \mathcal{U}^{(0)}\left(s_{1}, \boldsymbol{s}^{(0)}, s_{\mathrm{i}}\right) \times \\
& \mathcal{L}(\mathfrak{q}) \mathcal{L}\left(\mathfrak{q}_{p}\right) \cdots \mathcal{L}\left(\mathfrak{q}_{0}\right) \mathrm{d} \boldsymbol{s}^{(0)} \mathrm{d} \boldsymbol{s}^{(1)} \cdots \mathrm{d} \boldsymbol{s}^{(p-1)} \mathrm{d} \boldsymbol{s}^{(p)} \mathrm{d} s_{1} \cdots \mathrm{d} s_{p-1} \mathrm{~d} s_{p} \mathrm{~d} s_{p+1} \cdots \mathrm{d} s_{m-1} \mathrm{~d} s_{m},
\end{aligned}
$$

where the integrals with respect to $\boldsymbol{s}^{(k)}$ are interpreted by

$$
\int_{\boldsymbol{s}^{(k)} \subset(a, b)} \mathrm{d} \boldsymbol{s}^{(k)}=\int_{b>s_{n_{k}}^{(k)}>\cdots>s_{1}^{(k)}>a} \mathrm{~d} s_{1}^{(k)} \cdots \mathrm{d} s_{n_{k}}^{(k)} .
$$


Hence the integrals in the second line of (34) is actually the same as the integrals in (32). Due to the absolute convergence, we are allowed to safely interchange sums and integrals, which can give us the following factors:

$$
\begin{aligned}
& \sum_{n_{p}=0}^{+\infty} \int_{\boldsymbol{s}^{(p)} \subset\left(s_{p}, s_{\uparrow}\right)} \sum_{\mathfrak{q}_{p} \in \mathcal{Q}\left(\boldsymbol{s}^{(p)}\right)}(-1)^{\#\left\{\boldsymbol{s}^{(p)}<t\right\}} \mathrm{i}^{n_{k}} \mathcal{U}^{(0)}\left(s_{\uparrow}, \boldsymbol{s}^{(p)}, s_{p}\right) \mathcal{L}\left(\mathfrak{q}_{p}\right) \mathrm{d} \boldsymbol{s}^{(p)}, \\
& \sum_{n_{k}=0}^{+\infty} \int_{\boldsymbol{s}^{(k)} \subset\left(s_{k}, s_{k+1}\right)} \sum_{\mathfrak{q}_{k} \in \mathcal{Q}\left(\boldsymbol{s}^{(k)}\right)}(-1)^{\#\left\{\boldsymbol{s}^{(k)}<t\right\}^{n_{k}}} \mathcal{U}^{(0)}\left(s_{k+1}, \boldsymbol{s}^{(k)}, s_{k}\right) \mathcal{L}\left(\mathfrak{q}_{k}\right) \mathrm{d} \boldsymbol{s}^{(k)}, \quad k=0, \cdots, p-1 .
\end{aligned}
$$

This quantity can be replaced, respectively, by $G\left(s_{p}, s_{\uparrow}\right)$ and $G\left(s_{k+1}, s_{k}\right), k=0, \cdots, p-1$ according to (19). Such replacement turns (34) into

$$
\begin{gathered}
G\left(s_{\mathrm{f}}, s_{\mathrm{i}}\right)=G_{s}^{(0)}\left(s_{\mathrm{f}}, s_{\uparrow}\right) G\left(s_{\uparrow}, s_{\mathrm{i}}\right)+\sum_{\substack{m=2 \\
m \text { is even }}}^{+\infty} \sum_{p=0}^{m-1} \int_{s_{\mathrm{f}}>s_{m}>\cdots>s_{p+1}>s_{\uparrow}} \int_{s_{\uparrow}>s_{p}>\cdots>s_{1}>s_{\mathrm{i}}} \sum_{\mathfrak{q} \in \mathcal{Q}_{s_{\uparrow}}(\boldsymbol{s})}(-1)^{\#\{\boldsymbol{s}<t\}} \mathrm{i}^{m} \times \\
\mathcal{U}^{(0)}\left(s_{\mathrm{f}}, s_{m}, \cdots, s_{p+1}, s_{\uparrow}\right) G\left(s_{\uparrow}, s_{p}\right) W_{s} G\left(s_{p}, s_{p-1}\right) W_{s} \cdots W_{s} G\left(s_{2}, s_{1}\right) W_{s} G\left(s_{1}, s_{\mathrm{i}}\right) \mathcal{L}(\mathfrak{q}) \mathrm{d} s_{1} \cdots \mathrm{d} s_{p} \mathrm{~d} s_{p+1} \cdots \mathrm{d} s_{m} .
\end{gathered}
$$

Noting that

$$
\begin{aligned}
& \mathcal{U}^{(0)}\left(s_{\mathrm{f}}, s_{m}, \cdots, s_{p+1}, s_{\uparrow}\right) G\left(s_{\uparrow}, s_{p}\right) W_{s} G\left(s_{p}, s_{p-1}\right) W_{s} \cdots W_{s} G\left(s_{2}, s_{1}\right) W_{s} G\left(s_{1}, s_{\mathrm{i}}\right) \\
= & \mathcal{G}_{s_{\uparrow}}\left(s_{\mathrm{f}}, s_{m}\right) W_{s} \mathcal{G}_{s_{\uparrow}}\left(s_{m}, s_{m-1}\right) W_{s} \cdots W_{s} \mathcal{G}_{s_{\uparrow}}\left(s_{2}, s_{1}\right) W_{s} \mathcal{G}_{s_{\uparrow}}\left(s_{1}, s_{\mathrm{i}}\right),
\end{aligned}
$$

we see that the integrand in (37) is actually the same as the integrand in (23). Furthermore, the sum over $p$ in (37) can be replaced by $\sum_{p=0}^{m}$, because when $p=m$, the set $\mathcal{Q}_{s_{\uparrow}}(s)$ is empty. By doing this, we can apply the identity (27) and obtain the equality (23), which completes the proof of Theorem 1 .

Remark 3. In Theorem 1, when defining the absolute convergence, the absolute value is outside the sum of $\mathfrak{q}$ since $\mathcal{Q}(s)$ is a finite set. As mentioned in Remark 2, when the inchworm algorithm is applied to fermions, due to the switching sign in $\mathcal{L}(\mathfrak{q})$, the value of the sum over $\mathfrak{q}$ might become smaller due to cancellation; this would yield possibly improved convergence in bold diagrammatic Monte Carlo methods for fermionic systems.

\section{Integro-differential equations}

To better understand the algorithm, we are going to derive the limiting equation of the inchworm algorithm by considering the case in which $s_{\mathrm{f}}-s_{\uparrow}$ is infinitesimal. Suppose $\Delta t=s_{\mathrm{f}}-s_{\uparrow}$ and both $s_{\mathrm{f}}$ and $s_{\uparrow}$ are on the forward branch of the Keldysh contour, i.e. $s_{\uparrow}<s_{\mathrm{f}}<t$. By (7) and (9), we can find that

$$
\begin{gathered}
G_{s}^{(0)}\left(s_{\mathrm{f}}, s_{\uparrow}\right)=\mathrm{e}^{-\mathrm{i} \Delta t H_{s}}=I-\mathrm{i} \Delta t H_{s}+O\left(\Delta t^{2}\right), \\
\mathcal{U}^{(0)}\left(s_{\mathrm{f}}, s_{m}, s_{\uparrow}\right)=G_{s}^{(0)}\left(s_{\mathrm{f}}, s_{m}\right) W_{s} G_{s}^{(0)}\left(s_{m}, s_{\uparrow}\right)=W_{s}+O(\Delta t) .
\end{gathered}
$$

In the inchworm method (37), the domain of the integral

$$
\int_{s_{\mathrm{f}}>s_{m}>\cdots>s_{p+1}>s_{\uparrow}} \text { (integrand) } \mathrm{d} s_{p+1} \cdots \mathrm{d} s_{m-1} \mathrm{~d} s_{m}
$$


has volume $\frac{1}{(m-p) !} \Delta t^{m-p}$. Therefore all the terms with $p<m-1$ are higher order in $\Delta t$, and (37) can be rewritten as

$$
\begin{aligned}
G\left(s_{\mathrm{f}}, s_{\mathrm{i}}\right)= & G_{s}^{(0)}\left(s_{\mathrm{f}}, s_{\uparrow}\right) G\left(s_{\uparrow}, s_{\mathrm{i}}\right)+\sum_{\substack{m=2 \\
m \text { is even }}}^{+\infty} \mathrm{i}^{m} \int_{s_{\uparrow}}^{s_{\mathrm{f}}} \int_{s_{\uparrow}>s_{m-1}>\cdots>s_{1}>s_{\mathrm{i}}} \sum_{\mathfrak{q} \in \mathcal{Q}_{s_{\uparrow}}(\boldsymbol{s})}(-1)^{\#\{\boldsymbol{s}<t\}} \mathcal{L}(\mathfrak{q}) \times \\
& \mathcal{U}^{(0)}\left(s_{\mathrm{f}}, s_{m}, s_{\uparrow}\right) \mathcal{U}\left(s_{\uparrow}, s_{m-1}, \cdots, s_{1}, s_{\mathrm{i}}\right) \mathrm{d} s_{1} \cdots \mathrm{d} s_{m-1} \mathrm{~d} s_{m}+O\left(\Delta t^{2}\right) \\
= & \left(I-\mathrm{i} \Delta t H_{s}\right) G\left(s_{\uparrow}, s_{\mathrm{i}}\right)+\sum_{\substack{m=2 \\
m \text { is even }}}^{+\infty} \sum_{s_{\uparrow}}^{m} \int_{s_{\uparrow}>s_{m-1}>\cdots>s_{1}>s_{\mathrm{i}}}^{s_{\mathrm{f}} \in \mathcal{Q}_{s_{\uparrow}}(\boldsymbol{s})}(-1)^{\#\{\boldsymbol{s}<t\}} \mathcal{L}(\mathfrak{q}) \times \\
& W_{s} \mathcal{U}\left(s_{\uparrow}, s_{m-1}, \cdots, s_{1}, s_{\mathrm{i}}\right) \mathrm{d} s_{1} \cdots \mathrm{d} s_{m-1} \mathrm{~d} s_{m}+O\left(\Delta t^{2}\right),
\end{aligned}
$$

where we have used the short hand

$$
\mathcal{U}\left(s_{\uparrow}, s_{m-1}, \cdots, s_{1}, s_{\mathrm{i}}\right)=G\left(s_{\uparrow}, s_{m-1}\right) W_{s} G\left(s_{m-1}, s_{m-2}\right) W_{s} \cdots W_{s} G\left(s_{2}, s_{1}\right) W_{s} G\left(s_{1}, s_{\mathrm{i}}\right) .
$$

Our assumption that $s_{\uparrow}<s_{\mathrm{f}}<t$ shows that $\#\{s<t\}=m$. Also, by (10) and (21), one sees that

$$
\sum_{\mathfrak{q} \in \mathcal{Q}_{s_{\uparrow}}(\boldsymbol{s})} \mathcal{L}(\mathfrak{q})=\sum_{\mathfrak{q} \in \mathcal{Q}_{s_{\uparrow}}\left(s_{\uparrow}, s_{m}-1, \cdots, s_{1}\right)} \mathcal{L}(\mathfrak{q})+O(\Delta t)=\sum_{\mathfrak{q} \in \mathcal{Q}_{c}\left(s_{\uparrow}, s_{m}-1, \cdots, s_{1}\right)} \mathcal{L}(\mathfrak{q})+O(\Delta t) .
$$

Thus on the right-hand side of (39), the first-order term of the integrand is actually independent of $s_{m}$, which can then be integrated out. We write the result by moving $G\left(s_{\uparrow}, s_{\mathrm{i}}\right)$ to the left-hand side and divide both sides by $\Delta t$ :

$$
\begin{aligned}
\frac{G\left(s_{\mathrm{f}}, s_{\mathrm{i}}\right)-G\left(s_{\uparrow}, s_{\mathrm{i}}\right)}{\Delta t}=-\mathrm{i} H_{s} G\left(s_{\uparrow}, s_{\mathrm{i}}\right)-\sum_{\substack{m=2 \\
m \text { is even }}}^{+\infty} \mathrm{i}^{m} \int_{s_{\uparrow}>s_{m-1}>\cdots>s_{1}>s_{\mathrm{i}}} \\
\qquad \sum_{\mathfrak{q} \in \mathcal{Q}_{c}\left(s_{\uparrow}, s_{m-1}, \cdots, s_{1}\right)}(-1)^{m-1} \mathcal{L}(\mathfrak{q}) W_{s} \mathcal{U}\left(s_{\uparrow}, s_{m-1}, \cdots, s_{1}, s_{\mathrm{i}}\right) \mathrm{d} s_{1} \cdots \mathrm{d} s_{m-1}+O(\Delta t) .
\end{aligned}
$$

Taking the limit as $\Delta t \rightarrow 0$ and renaming $m$ to $m+1$, we obtain the integro-differential equations for the propagator $G(\cdot, \cdot)$ :

$$
\begin{aligned}
\frac{\partial G\left(s_{\uparrow}, s_{\mathrm{i}}\right)}{\partial s_{\uparrow}}= & -\mathrm{i} H_{s} G\left(s_{\uparrow}, s_{\mathrm{i}}\right) \\
& -\sum_{\substack{m=1 \\
m \text { is odd }}}^{+\infty} \mathrm{i}^{m+1} \int_{s_{\uparrow}>s_{m}>\cdots>s_{1}>s_{\mathrm{i}}} \sum_{\mathfrak{q} \in \mathcal{Q}_{c}\left(s_{\uparrow}, \boldsymbol{s}\right)}(-1)^{\#\{\boldsymbol{s}<t\}} \mathcal{L}(\mathfrak{q}) W_{s} \mathcal{U}\left(s_{\uparrow}, \boldsymbol{s}, s_{\mathrm{i}}\right) \mathrm{d} s_{1} \cdots \mathrm{d} s_{m} .
\end{aligned}
$$

Here we have again used the fact that all the components of $s$ are less than $t$. The equation (41) gives an integro-differential equation for the full propagator $G\left(s_{\uparrow}, s_{\mathrm{i}}\right)$ when $s_{\uparrow}<t$. If $s_{\uparrow}>t$, we can use the same method to derive a similar integro-differential equation:

$$
\begin{aligned}
\frac{\partial G\left(s_{\uparrow}, s_{\mathrm{i}}\right)}{\partial s_{\uparrow}} & =\mathrm{i} H_{s} G\left(s_{\uparrow}, s_{\mathrm{i}}\right) \\
& +\sum_{\substack{m=1 \\
m \text { is odd }}}^{+\infty} \mathrm{i}^{m+1} \int_{s_{\uparrow}>s_{m}>\cdots>s_{1}>s_{\mathrm{i}}} \sum_{\mathfrak{q} \in \mathcal{Q}_{c}\left(s_{\uparrow}, \boldsymbol{s}\right)}(-1)^{\#\{\boldsymbol{s}<t\}} \mathcal{L}(\mathfrak{q}) W_{s} \mathcal{U}\left(s_{\uparrow}, \boldsymbol{s}, s_{\mathrm{i}}\right) \mathrm{d} s_{1} \cdots \mathrm{d} s_{m} .
\end{aligned}
$$

When $s_{\uparrow}=t$, one can see from (13) that $G\left(\cdot, s_{\mathrm{i}}\right)$ is discontinuous, and it satisfies

$$
\lim _{s_{\uparrow} \rightarrow t^{+}} G\left(s_{\uparrow}, s_{\mathrm{i}}\right)=O_{s} \lim _{s_{\uparrow} \rightarrow t^{-}} G\left(s_{\uparrow}, s_{\mathrm{i}}\right) .
$$

Combing (41) and (42), we get the following theorem: 
Theorem 2. When the Dyson series (19) is absolutely convergent in the sense of (22), the full propagator $G(\cdot, \cdot)$ satisfies the integro-differential equation

$$
\begin{aligned}
\operatorname{sgn}\left(s_{\uparrow}-t\right) \frac{\partial G\left(s_{\uparrow}, s_{\mathrm{i}}\right)}{\partial s_{\uparrow}} & =\mathrm{i} H_{s} G\left(s_{\uparrow}, s_{\mathrm{i}}\right)+\sum_{\substack{m=1 \\
m \text { is odd }}}^{+\infty} \mathrm{i}^{m+1} \int_{s_{\uparrow}>s_{m}>\cdots>s_{1}>s_{\mathrm{i}}} \\
& \sum_{\mathfrak{q} \in \mathcal{Q}_{c}\left(s_{\uparrow}, s\right)}(-1)^{\#\{\boldsymbol{s}<t\}} \mathcal{L}(\mathfrak{q}) W_{s} \mathcal{U}\left(s_{\uparrow}, \boldsymbol{s}, s_{\mathrm{i}}\right) \mathrm{d} s_{1} \cdots \mathrm{d} s_{m}, \quad \forall s_{\mathrm{i}} \in[0,2 t] \backslash\{t\}, \quad s_{\uparrow} \in\left[s_{\mathrm{i}}, 2 t\right] \backslash\{t\},
\end{aligned}
$$

with the jump condition (43) and the "initial condition" $G\left(s_{\uparrow}, s_{\uparrow}\right)=\mathrm{Id}$.

Although the integro-differential equation (44) has been derived from the inchworm method, the infinitesimal terms $O(\Delta t)$ or $O\left(\Delta t^{2}\right)$ have not been rigorously verified. Below we are going to provide a rigorous proof of Theorem 2 starting from the definition of $G(\cdot, \cdot)$.

Proof of Theorem 圆. We will start the proof by deriving the dynamics of the propagator with a more straightforward method, and then show that the result is equivalent to (44). Again we consider the case $s_{\uparrow}<t$, and take derivative of the definition of $G$ (13) to get

$$
\begin{aligned}
\frac{\partial G\left(s_{\uparrow}, s_{\mathrm{i}}\right)}{\partial s_{\uparrow}} & =\operatorname{tr}_{b}\left(\rho_{b} G_{b}^{(0)}\left(2 t, s_{\uparrow}\right)\left(\mathrm{i} H_{b}-\mathrm{i} H\right) \mathrm{e}^{-\mathrm{i}\left(s_{\uparrow}-s_{\mathrm{i}}\right) H} G_{b}^{(0)}\left(s_{\mathrm{i}}, 0\right)\right) \\
& =-\mathrm{i} \operatorname{tr}_{b}\left(\rho_{b} G_{b}^{(0)}\left(2 t, s_{\uparrow}\right)\left(H_{s}+W\right) \mathrm{e}^{-\mathrm{i}\left(s_{\uparrow}-s_{\mathrm{i}}\right) H} G_{b}^{(0)}\left(s_{\mathrm{i}}, 0\right)\right) \\
& =-\mathrm{i} H_{s} G\left(s_{\uparrow}, s_{\mathrm{i}}\right)-\mathrm{i} W_{s} \operatorname{tr}_{b}\left(\rho_{b} G_{b}^{(0)}\left(2 t, s_{\uparrow}\right) W_{b} \mathrm{e}^{-\mathrm{i}\left(s_{\uparrow}-s_{\mathrm{i}}\right) H} G_{b}^{(0)}\left(s_{\mathrm{i}}, 0\right)\right) .
\end{aligned}
$$

The propagator $\mathrm{e}^{-\mathrm{i}\left(s_{\uparrow}-s_{\mathrm{i}}\right) H}$ can be expanded into Dyson series as (3), which turns (45) into

$$
\begin{aligned}
& \frac{\partial G\left(s_{\uparrow}, s_{\mathrm{i}}\right)}{\partial s_{\uparrow}}=-\mathrm{i} H_{s} G\left(s_{\uparrow}, s_{\mathrm{i}}\right)-\sum_{m=0}^{+\infty} \mathrm{i}^{m+1} \int_{s_{\uparrow}>s_{m}>\cdots>s_{1}>s_{\mathrm{i}}} \\
& (-1)^{m} W_{s} G_{s}^{(0)}\left(s_{\uparrow}, s_{m}\right) W_{s} G_{s}^{(0)}\left(s_{m}, s_{m-1}\right) W_{s} \cdots W_{s} G_{s}^{(0)}\left(s_{2}, s_{1}\right) W_{s} G_{s}^{(0)}\left(s_{1}, s_{\mathrm{i}}\right) \times \\
& \operatorname{tr}_{b}\left(\rho_{b} G_{b}^{(0)}\left(2 t, s_{\uparrow}\right) W_{b} G_{b}^{(0)}\left(s_{\uparrow}, s_{m}\right) W_{b} G_{b}^{(0)}\left(s_{m}, s_{m-1}\right) W_{b} \cdots W_{b} G_{b}^{(0)}\left(s_{2}, s_{1}\right) W_{b} G_{b}^{(0)}\left(s_{1}, s_{\mathrm{i}}\right) G_{b}^{(0)}\left(s_{\mathrm{i}}, 0\right)\right) \\
& \mathrm{d} s_{1} \cdots \mathrm{d} s_{m} .
\end{aligned}
$$

Since $G_{b}^{(0)}\left(s_{1}, s_{\mathrm{i}}\right) G_{b}^{(0)}\left(s_{\mathrm{i}}, 0\right)=G_{b}^{(0)}\left(s_{1}, 0\right)$, we can use the definitions (10) and (9) to simplify the above equation:

$$
\begin{aligned}
& \frac{\partial G\left(s_{\uparrow}, s_{\mathrm{i}}\right)}{\partial s_{\uparrow}}=-\mathrm{i} H_{s} G\left(s_{\uparrow}, s_{\mathrm{i}}\right)-\sum_{m=0}^{+\infty} \mathrm{i}^{m+1} \int_{s_{\uparrow}>s_{m}>\cdots>s_{1}>s_{\mathrm{i}}}(-1)^{m} W_{s} \mathcal{U}^{(0)}\left(s_{\uparrow}, \boldsymbol{s}, s_{\mathrm{i}}\right) \mathcal{L}\left(s_{\uparrow}, \boldsymbol{s}\right) \mathrm{d} s_{1} \cdots \mathrm{d} s_{m} \\
& =-\mathrm{i} H_{s} G\left(s_{\uparrow}, s_{\mathrm{i}}\right)-\sum_{\substack{m=1 \\
m \text { is odd }}}^{+\infty} \mathrm{i}^{m+1} \int_{s_{\uparrow}>s_{m}>\cdots>s_{1}>s_{\mathrm{i}}} \sum_{\mathfrak{q} \in \mathcal{Q}_{c}(\boldsymbol{s})}(-1)^{\#\{\boldsymbol{s}<t\}} W_{s} \mathcal{U}^{(0)}\left(s_{\uparrow}, \boldsymbol{s}, s_{\mathrm{i}}\right) \mathcal{L}(\mathfrak{q}) \mathrm{d} s_{1} \cdots \mathrm{d} s_{m} .
\end{aligned}
$$

To see that the above equation is identical to the "inchworm equation" (41), we can mimic (34) and replace the propagators $G(\cdot, \cdot)$ in the integral of (41) by its Dyson series expansion. Following the same way as in the previous section, we obtain a result similar to (29):

$$
\begin{aligned}
\frac{\partial G\left(s_{\uparrow}, s_{\mathrm{i}}\right)}{\partial s_{\uparrow}}= & -\mathrm{i} H_{s} G\left(s_{\uparrow}, s_{\mathrm{i}}\right)-\sum_{\substack{m=1 \\
m \text { is odd }}}^{+\infty} \sum_{\substack{n_{m}=0 \\
n_{m} \text { is even }}}^{+\infty} \ldots \sum_{\substack{n_{0}=0 \\
n_{0} \text { is even }}}^{+\infty} \mathrm{i}^{\tilde{m}+1} \int_{s_{\uparrow}>\tilde{s}_{\tilde{m}}>\cdots>\tilde{s}_{1}>s_{\mathrm{i}}} \\
& \sum_{\mathfrak{q} \in \mathcal{Q}_{c}\left(s_{\uparrow}, \boldsymbol{s}\right)} \sum_{\mathfrak{q}_{m} \in \mathcal{Q}\left(\boldsymbol{s}^{(m)}\right)} \cdots \sum_{\mathfrak{q}_{0} \in \mathcal{Q}\left(\boldsymbol{s}^{(0)}\right)}(-1)^{\#\{\tilde{\boldsymbol{s}}<t\}} W_{s} \mathcal{U}^{(0)}\left(s_{\uparrow}, \tilde{\boldsymbol{s}}, s_{\mathrm{i}}\right) \mathcal{L}(\tilde{\mathfrak{q}}) \mathrm{d} \tilde{s}_{1} \cdots \mathrm{d} \tilde{s}_{\tilde{m}},
\end{aligned}
$$


where

$$
\tilde{\mathfrak{q}}=\mathfrak{q} \cup \mathfrak{q}_{m} \cup \cdots \cup \mathfrak{q}_{0}, \quad \tilde{m}=m+n_{m}+\cdots+n_{0}, \quad \tilde{\boldsymbol{s}}=\left(\tilde{s}_{\tilde{m}}, \cdots, \tilde{s}_{1}\right),
$$

and $\boldsymbol{s}, \boldsymbol{s}^{(m)}, \cdots, \boldsymbol{s}^{(0)}$ can be determined by

$$
\tilde{\boldsymbol{s}}=\left(\boldsymbol{s}^{(m)}, s_{m}, \boldsymbol{s}^{(m-1)}, s_{m-1}, \cdots, \boldsymbol{s}^{(1)}, s_{1}, \boldsymbol{s}^{(0)}\right), \quad \boldsymbol{s}=\left(s_{m}, \cdots, s_{1}\right) .
$$

Now we need to use the following equivalence of sums:

$$
\sum_{\substack{m=1 \\ m \text { is odd }}}^{+\infty} \sum_{\substack{n_{m}=0 \\ n_{m} \text { is even }}}^{+\infty} \cdots \sum_{\substack{n_{0}=0 \\ n_{0} \text { is even }}}^{+\infty}=\sum_{\substack{\tilde{m}=1 \\ \tilde{m} \text { is odd } \tilde{m}-m \text { is even }}}^{+\infty} \sum_{\substack{n_{m}=0 \\ n_{m} \text { is even }}}^{\tilde{m}} \cdots \sum_{\substack{n_{m-1}=0 \\ n_{m-1} \text { is even }}}^{\tilde{m}-m} \cdots \sum_{\substack{n_{1}=0 \\ n_{1} \text { is even }}}^{\tilde{m}-m-n_{m}} \ldots
$$

Due to the absolute convergence of the Dyson series, the equation (48) becomes

$$
\begin{aligned}
& \frac{\partial G\left(s_{\uparrow}, s_{\mathrm{i}}\right)}{\partial s_{\uparrow}}=-\mathrm{i} H_{s} G\left(s_{\uparrow}, s_{\mathrm{i}}\right)-\sum_{\substack{\tilde{m}=1 \\
\tilde{m} \text { is odd }}}^{+\infty} \mathrm{i}^{\tilde{m}+1} \int_{s_{\uparrow}>\tilde{s}_{\tilde{m}}>\cdots>\tilde{s}_{1}>s_{\mathrm{i}}} \\
& \sum_{\substack{m=1 \\
\tilde{m}-m}}^{\tilde{m} \text { is even }} \sum_{\substack{n_{m}=0 \\
n_{m} \text { is even }}}^{\tilde{m}-m} \sum_{n_{m-1} \text { is even }}^{\tilde{m}-m-n_{m}} \cdots \sum_{\substack{n_{1}=0 \\
n_{1} \text { is even }}}^{\tilde{m}-m-n_{m}-\cdots-n_{2}} \sum_{\mathfrak{q} \in \mathcal{Q}_{c}\left(s_{\uparrow}, \boldsymbol{s}\right)} \sum_{\mathfrak{q}_{m} \in \mathcal{Q}\left(\boldsymbol{s}^{(m)}\right)} \cdots \sum_{\mathfrak{q}_{0} \in \mathcal{Q}\left(\boldsymbol{s}^{(0)}\right)} \\
& (-1)^{\#\{\tilde{\boldsymbol{s}}<t\}} W_{s} \mathcal{U}^{(0)}\left(s_{\uparrow}, \tilde{\boldsymbol{s}}, s_{\mathrm{i}}\right) \mathcal{L}(\tilde{\mathfrak{q}}) \mathrm{d} \tilde{s}_{1} \cdots \mathrm{d} \tilde{s}_{\tilde{m}} .
\end{aligned}
$$

The last step is to verify that the right-hand side of the above equation equals the right-hand side of (47). By comparison, we just need to show that for any given positive odd integer $\tilde{m}$ and a sequence $\tilde{\boldsymbol{s}}=\left(s_{\tilde{m}}, \cdots, s_{1}\right)$ satisfying $s_{\uparrow}>s_{\tilde{m}}>\cdots>s_{1}>s_{\mathrm{i}}$, the following map is a bijection:

$$
\begin{array}{ccc}
\widetilde{\mathcal{P}} & \rightarrow & \mathcal{Q}_{s_{\uparrow}}(\tilde{s}) \\
\left(m, n_{m}, \cdots, n_{0}, \mathfrak{q}, \mathfrak{q}_{p}, \cdots, \mathfrak{q}_{0}\right) & \mapsto \mathfrak{q} \cup \mathfrak{q}_{p} \cup \cdots \cup \mathfrak{q}_{0}
\end{array}
$$

where

$$
\begin{aligned}
\widetilde{\mathcal{P}}=\left\{\left(m, n_{m}, \cdots, n_{0}, \mathfrak{q}, \mathfrak{q}_{p}, \cdots, \mathfrak{q}_{0}\right) \mid p\right. & \in\{\tilde{m}, \tilde{m}-2, \cdots, 1\} ; n_{0}+\cdots+n_{m}=\tilde{m}-m ; n_{m}, \cdots, n_{0} \text { are even; } \\
\mathfrak{q} & \left.\in \mathcal{Q}_{c}\left(s_{\uparrow}, \boldsymbol{s}\right) ; \mathfrak{q}_{m} \in \mathcal{Q}\left(\boldsymbol{s}^{(p)}\right) ; \cdots ; \mathfrak{q}_{0} \in \mathcal{Q}\left(\boldsymbol{s}^{(0)}\right)\right\}
\end{aligned}
$$

This map can actually be considered as a special case of (30) when $\tilde{p}=\tilde{m}-1$, and hence is one-to-one. Till now, the inchworm equation (44) is confirmed to be identical to (45) when $s_{\uparrow}<t$, which describes the correct dynamics of the full propagator. The case $s_{\uparrow}>t$ can be dealt with following the same procedure, which is omitted for conciseness.

Since the equation in the above theorem is firstly derived by setting $s_{\mathrm{f}}-s_{\uparrow}$ to be infinitesimal in the inchworm algorithm, the algorithm can actually be considered as an iterative scheme for the equation. From this point of view, we can improve the numerical method by solving (44) with a higher-order scheme. Before introducing the details of the method, we will first present the spin-boson model, and show that it satisfies all the conditions needed for the inchworm method.

Remark 4. The sum of integrals on the right-hand side of Eq. (44), can be rewritten as

$$
\int_{s_{\mathrm{i}}}^{s_{\uparrow}} \Sigma\left(s_{\uparrow}, s_{1}\right) G\left(s_{1}, s_{\mathrm{i}}\right) \mathrm{d} s_{1}
$$

with a properly defined $\Sigma\left(s_{\uparrow}, s_{1}\right)$, which makes the equation (44) similar to the Kadanoff-Baym equations [26]. It would be interesting to establish deeper connections between these models, which we will leave for future works. 


\section{Spin-boson model}

To demonstrate the algorithm in a specific model, we consider the spin-boson model in which the system is a single spin and the bath is given by a large number of harmonic oscillators. In detail, we have

$$
\mathcal{H}_{s}=\operatorname{span}\{|1\rangle,|2\rangle\}, \quad \mathcal{H}_{b}=\bigotimes_{l=1}^{L}\left(L^{2}\left(\mathbb{R}^{3}\right)\right)
$$

where $L$ is the number of harmonic oscillators. The corresponding Hamiltonians are

$$
H_{s}=\epsilon \hat{\sigma}_{z}+\Delta \hat{\sigma}_{x}, \quad H_{b}=\sum_{l=1}^{L} \frac{1}{2}\left(\hat{p}_{l}^{2}+\omega_{l}^{2} \hat{q}_{l}^{2}\right) .
$$

The notations are described as follows:

- $\epsilon$ : energy difference between two spin states.

- $\Delta$ : frequency of the spin flipping.

- $\hat{\sigma}_{x}, \hat{\sigma}_{z}$ : Pauli matrices satisfying $\hat{\sigma}_{x}|1\rangle=|2\rangle, \hat{\sigma}_{x}|2\rangle=|1\rangle, \hat{\sigma}_{z}|1\rangle=|1\rangle, \hat{\sigma}_{z}|2\rangle=-|2\rangle$.

- $\omega_{l}$ : frequency of the $l$ th harmonic oscillator.

- $\hat{q}_{l}$ : position operator for the $l$ th harmonic oscillator defined by $\psi\left(q_{1}, \cdots, q_{L}\right) \mapsto q_{l} \psi\left(q_{1}, \cdots, q_{L}\right)$.

- $\hat{p}_{l}$ : momentum operator for the $l$ th harmonic oscillator defined by $\psi\left(q_{1}, \cdots, q_{L}\right) \mapsto-\mathrm{i} \nabla_{q_{l}} \psi\left(q_{1}, \cdots, q_{L}\right)$.

The coupling between system and bath is assumed to be linear:

$$
W=W_{s} \otimes W_{b}, \quad W_{s}=\hat{\sigma}_{z}, \quad W_{b}=\sum_{l=1}^{L} c_{l} \hat{q}_{l},
$$

where $c_{l}$ is the coupling intensity between the $l$ th harmonic oscillator and the spin. Suppose the initial state of the bath is in the thermal equilibrium with inverse temperature $\beta$, i.e. $\rho_{b}=Z^{-1} \exp \left(-\beta H_{b}\right)$, and $Z$ is chosen such that $\operatorname{tr}\left(\rho_{b}\right)=1$. Thus the hypothesis (H1) is fulfilled, and Wick's theorem (15) holds for

$$
B\left(\tau_{1}, \tau_{2}\right)=\sum_{l=1}^{L} \frac{c_{l}^{2}}{2 \omega_{l}}\left[\operatorname{coth}\left(\frac{\beta \omega_{l}}{2}\right) \cos \omega_{l}\left(\tau_{2}-\tau_{1}\right)-\mathrm{i} \sin \omega_{l}\left(\tau_{2}-\tau_{1}\right)\right] .
$$

In order to apply the inchworm algorithm to the spin-boson model, we need to show the absolute convergence (22). By the definition of $\mathcal{U}(9)$, we immediately have

$$
\left\|\mathcal{U}\left(s_{\mathrm{f}}, s, s_{\mathrm{i}}\right)\right\|_{s} \leqslant\left\|W_{s}\right\|_{s}^{m} \max \left\{\left\|O_{s}\right\|_{s}, 1\right\}=\left\|\hat{\sigma}_{z}\right\|_{s}^{m} \max \left\{\left\|O_{s}\right\|_{s}, 1\right\}=\max \left\{\left\|O_{s}\right\|_{s}, 1\right\} .
$$

And from (51), we see that

$$
\left|B\left(\tau_{1}, \tau_{2}\right)\right| \leqslant \sum_{l=1}^{L} \frac{c_{l}^{2}}{2 \omega_{l}} \sqrt{\operatorname{coth}^{2}\left(\frac{\beta \omega_{l}}{2}\right) \cos ^{2} \omega_{l}\left(\tau_{2}-\tau_{1}\right)+\sin ^{2} \omega_{l}\left(\tau_{2}-\tau_{1}\right)} \leqslant \sum_{l=1}^{L} \frac{c_{l}^{2}}{2 \omega_{l}} \operatorname{coth}\left(\frac{\beta \omega_{l}}{2}\right) .
$$

Let $C_{b}$ be the right-hand side of the above inequality. Then when $m$ is even,

$$
|\mathcal{L}(\mathfrak{q})| \leqslant C_{b}^{m / 2}, \quad \forall \mathfrak{q} \in \mathcal{Q}\left(s_{m}, \cdots, s_{1}\right) .
$$


Since the number of pair sets in $\mathcal{Q}\left(s_{m}, \cdots, s_{1}\right)$ is $(m-1) !$ !, we have

$$
\begin{aligned}
& \sum_{\substack{m=0 \\
\text { is even }}}^{+\infty} \int_{s_{\mathrm{f}}>s_{m}>\cdots>s_{1}>s_{\mathrm{i}}}\left\|\mathcal{U}^{(0)}\left(s_{\mathrm{f}}, \boldsymbol{s}, s_{\mathrm{i}}\right)\right\|_{s}\left|\sum_{\mathfrak{q} \in \mathcal{Q}(\boldsymbol{s})} \mathcal{L}(\mathfrak{q})\right| \mathrm{d} s_{1} \cdots \mathrm{d} s_{m} \\
\leqslant & \sum_{\substack{m=0 \\
m \text { is even }}}^{+\infty} \int_{s_{\mathrm{f}}>s_{m}>\cdots>s_{1}>s_{\mathrm{i}}} \max \left\{\left\|O_{s}\right\|_{s}, 1\right\} \cdot(m-1) ! ! C_{b}^{m / 2} \mathrm{~d} s_{1} \cdots \mathrm{d} s_{m} \\
= & \max \left\{\left\|O_{s}\right\|_{s}, 1\right\} \sum_{\substack{m=0 \\
m \text { is even }}}^{+\infty} \frac{\left(s_{\mathrm{f}}-s_{\mathrm{i}}\right)^{m}}{m ! !} C_{b}^{m / 2}=\max \left\{\left\|O_{s}\right\|_{s}, 1\right\} \exp \left(\frac{C_{b}\left(s_{\mathrm{f}}-s_{\mathrm{i}}\right)^{2}}{2}\right) .
\end{aligned}
$$

Since $\mathcal{H}_{s}$ is a finite-dimensional space, the observable $O_{s}$ is always a bounded operator. Therefore the righthand side of the above equation is finite, which shows the absolute convergence. In the spin-boson model, people are usually interested in the population of the spin on each of the two spin states, meaning that we can take $O_{s}=\hat{\sigma}_{z}$.

When the Dyson series expansion (19) is directly used in the Monte Carlo simulation, the fast growth of the variance as $s_{\mathrm{f}}-s_{\mathrm{i}}$ increases causes great numerical difficulties. Similar to (53), the expectation of $\left\|\mathcal{U}^{(0)}\left(s_{\mathrm{f}}, \boldsymbol{s}, s_{\mathrm{i}}\right) \mathcal{L}(\mathfrak{q})\right\|_{s}^{2}$ can be estimated by

$$
\begin{aligned}
& \sum_{\substack{m=0 \\
m \text { is even }}}^{+\infty} \int_{s_{\mathrm{f}}>s_{m}>\cdots>s_{1}>s_{\mathrm{i}}} \sum_{\mathfrak{q} \in \mathcal{Q}(\boldsymbol{s})}\left\|\mathcal{U}^{(0)}\left(s_{\mathrm{f}}, \boldsymbol{s}, s_{\mathrm{i}}\right)\right\|_{s}^{2}|\mathcal{L}(\mathfrak{q})|^{2} \mathrm{~d} s_{1} \cdots \mathrm{d} s_{m} \\
\leqslant & \sum_{\substack{m=0 \\
m \text { is even }}}^{+\infty} \int_{s_{\mathrm{f}}>s_{m}>\cdots>s_{1}>s_{\mathrm{i}}}(m-1) ! !\left(\max \left\{\left\|O_{s}\right\|_{s}, 1\right\}\right)^{2} C_{b}^{m} \mathrm{~d} s_{1} \cdots \mathrm{d} s_{m} \\
= & \left(\max \left\{\left\|O_{s}\right\|_{s}, 1\right\}\right)^{2} \exp \left(\frac{C_{b}^{2}\left(s_{\mathrm{f}}-s_{\mathrm{i}}\right)^{2}}{2}\right) .
\end{aligned}
$$

Since $\left\|G\left(s_{\mathrm{f}}, s_{\mathrm{i}}\right)\right\|_{s} \leqslant \max \left\{\left\|O_{s}\right\|_{s}, 1\right\}$, the growth of the variance is characterized by

$$
\exp \left(\frac{C_{b}^{2}\left(s_{\mathrm{f}}-s_{\mathrm{i}}\right)^{2}}{2}\right)-1 .
$$

This is the well-known "dynamical sign problem" in the quantum Monte Carlo simulations. The inchworm method relieves the dynamical sign problem by lumping a number of samples based on the simulation results of shorter bold lines, which pushes the simulation time significantly longer.

Different from the inchworm methods presented in [4], which directly applies the Monte Carlo method to (23), we will design our numerical method based on the integro-differential equation (44), and apply the idea of classical Runge-Kutta type methods for temporal discretization to solve the full propagators. As will be presented, this can both enhance the numerical efficiency and simplify the implementation. The algorithm will be detailed in the following section.

\section{Numerical method}

In order to find the full propagator $G\left(s_{\mathrm{f}}, s_{\mathrm{i}}\right)$ numerically for all $s_{\mathrm{i}} \in[0,2 t]$ and $s_{\mathrm{f}} \in\left[s_{\mathrm{i}}, 2 t\right]$, below we are going to develop a second-order method in analogous to Heun's method for general ordinary differential equations. For the general initial value problem

$$
\frac{\mathrm{d}}{\mathrm{d} t} u(t)=f(t, u(t)), \quad t>0
$$


with initial condition $u(0)=u_{0}$, Heun's method reads

$$
\begin{aligned}
U_{k}^{*} & =U_{k-1}+\Delta t f\left(t_{k-1}, U_{k-1}\right), \\
U_{k} & =\frac{1}{2}\left(U_{k-1}+U_{k}^{*}\right)+\frac{1}{2} \Delta t f\left(t_{k}, U_{k}^{*}\right),
\end{aligned}
$$

where $\Delta t$ is the time step, $t_{k}=t_{k-1}+\Delta t$, and $U_{k}$ is the numerical approximation of $u\left(t_{k}\right)$. The method is second-order if the solution is third-order continuously differentiable. In our case, the full propagator $G(\cdot, \cdot)$ is known to be discontinuous on the line segments $[0, t] \times\{t\}$ and $\{t\} \times[0, t]$ due to the presence of the observable $O_{s}$. Therefore in order to keep the second-order convergence rate, special care needs to be taken for these discontinuities.

In our implementation, we take a uniform time step $\Delta t=t / N$, and compute the numerical solutions $G\left(s_{\mathrm{f}}, s_{\mathrm{i}}\right)$ only when $s_{\mathrm{f}}$ and $s_{\mathrm{i}}$ are multiples of $\Delta t$. This corresponds to a two-dimensional triangular mesh shown in Figure 4 (for $N=5$ ). Let $t_{k}=k \Delta t$ and $G_{j k}^{\Delta t}$ be the numerical approximation of $G\left(t_{j}, t_{k}\right)$. Due to the discontinuity, when $j=N$ (green line in Figure 4) or $k=N$ (blue line in Figure 4), $G_{j k}^{\Delta t}$ is considered to be multiple-valued, and we will use the notation $j, k=N^{+}, N^{-}$to define the left and right limits. Precisely,

- For $k<N, G_{N^{-} k}^{\Delta t}$ and $G_{N^{+} k}^{\Delta t}$ are respectively the approximation of $\lim _{s \rightarrow t^{-}} G(s, k \Delta t)$ and $\lim _{s \rightarrow t^{+}} G(s, k \Delta t)$.

- For $j>N, G_{j N^{-}}^{\Delta t}$ and $G_{j N^{+}}^{\Delta t}$ are respectively the approximation of $\lim _{s \rightarrow t^{-}} G(j \Delta t, s)$ and $\lim _{s \rightarrow t^{+}} G(j \Delta t, s)$.

- $G_{N^{-} N^{-}}^{\Delta t}=G_{N^{+} N^{+}}^{\Delta t}=\operatorname{Id}$ and $G_{N^{+} N^{-}}^{\Delta t}=O_{s}$.

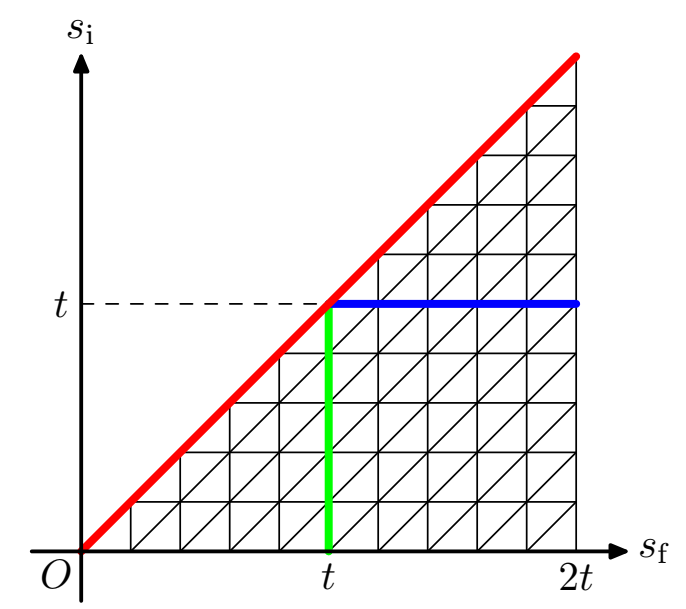

Figure 4: The two-dimensional mesh to discretize $G\left(s_{\mathrm{f}}, s_{\mathrm{i}}\right)$ for $N=5$

Now we are ready to sketch our numerical algorithm. In general, the values of $G_{j k}^{\Delta t}$ are obtained in the 
following order:

$$
\begin{aligned}
& G_{00}^{\Delta t} \\
& G_{11}^{\Delta t}, \quad G_{10}^{\Delta t}, \\
& \text { ․ } \quad \cdots \quad \cdots \quad \cdots \\
& G_{N-1, N-1}^{\Delta t}, \quad \cdots, \quad G_{N-1,0}^{\Delta t}, \\
& G_{N^{-}, N^{-}}^{\Delta t}, \quad G_{N^{-}, N-1}^{\Delta t}, \quad \cdots, \quad G_{N^{-}, 0}^{\Delta t}, \\
& G_{N^{+}, N^{+}}^{\Delta t}, \quad G_{N^{+}, N^{-}}^{\Delta t}, \quad G_{N^{+}, N-1}^{\Delta t}, \quad \cdots, \quad G_{N^{+}, 0}^{\Delta t}, \\
& G_{N+1, N+1}^{\Delta t}, \quad G_{N+1, N^{+}}^{\Delta t}, \quad G_{N+1, N^{-}}^{\Delta t}, \quad G_{N+1, N-1}^{\Delta t}, \quad \cdots, \quad G_{N+1,0}^{\Delta t}, \\
& \begin{array}{llllllllllllll}
\ldots & \ldots & \ldots & \ldots & \ldots & \ldots & \ldots & \ldots & \ldots & \ldots & \ldots & \ldots & \ldots & \ldots
\end{array} \\
& G_{2 N, 2 N}^{\Delta t}, \quad \cdots, \quad G_{2 N, N+1}^{\Delta t}, \quad G_{2 N, N^{+}}^{\Delta t}, \quad G_{2 N, N^{-}}^{\Delta t}, \quad G_{2 N, N-1}^{\Delta t}, \quad \cdots, \quad G_{2 N, 0}^{\Delta t} .
\end{aligned}
$$

This corresponds to computing the values of $G(\cdot, \cdot)$ column by column in Figure 4 with the green line and the blue line split to two lines due to the discontinuity. We first list out the three special cases:

- If $j=k\left(N^{+}\right.$is considered to be not equal to $\left.N^{-}\right)$, we set $G_{j k}^{\Delta t}$ to be Id. This corresponds to the nodes on the red line in Figure 4.

- If $j=N^{+}$and $k \neq j$, we set $G_{j k}^{\Delta t}$ to be $O_{s} G_{N^{-} k}^{\Delta t}$. This is applied to the nodes on the green line in Figure 4

- If $k=N^{-}$and $j \neq k$, we set $G_{j k}^{\Delta t}$ to be $G_{j N^{+}}^{\Delta t} O_{s}$. This is applied to the nodes on the blue line in Figure 4 .

For all other cases, we follow Heun's method and find the values of $G_{j k}^{\Delta t}$ as follows:

1. Let

$$
\begin{aligned}
G_{j k}^{*}=G_{j-1, k}^{\Delta t} & +\operatorname{sgn}\left(t_{j}-t\right) \Delta t\left[\mathrm{i} H_{s} G_{j-1, k}^{\Delta t}+\sum_{\substack{m=1 \\
m \text { is odd }}}^{+\infty} \mathrm{i}^{m+1} \int_{t_{j-1}>s_{m}>\cdots>s_{1}>t_{k}} \sum_{\mathfrak{q} \in \mathcal{Q}_{c}\left(t_{j-1}, \boldsymbol{s}\right)}(-1)^{\#\{\boldsymbol{s}<t\}}\right. \\
& \left.\times \mathcal{L}(\mathfrak{q}) W_{s} G_{I}\left(t_{j-1}, s_{m}\right) W_{s} G_{I}\left(s_{m}, s_{m-1}\right) W_{s} \cdots W_{s} G_{I}\left(s_{2}, s_{1}\right) W_{s} G_{I}\left(s_{1}, t_{k}\right) \mathrm{d} s_{1} \cdots \mathrm{d} s_{m}\right],
\end{aligned}
$$

where $G_{I}(\cdot, \cdot)$ is the interpolated function satisfying

$$
G_{I}\left(t_{j^{\prime}}, t_{k^{\prime}}\right)=G_{j^{\prime} k^{\prime}}^{\Delta t}, \quad \text { for all integers } j^{\prime}, k^{\prime} \text { satisfying } k \leqslant k^{\prime} \leqslant j^{\prime} \leqslant j-1 .
$$

In our implementation, piecewise linear interpolation is adopted, and the function $G_{I}(\cdot, \cdot)$ is linear on each triangle in Figure 4 .

2. Set $G_{j k}^{\Delta t}$ to be

$$
\begin{aligned}
\frac{1}{2} G_{j-1, k}^{\Delta t}+\frac{1}{2} G_{j k}^{*}+\frac{1}{2} \operatorname{sgn}\left(t_{j}-t\right) \Delta t\left[\mathrm{i} H_{s} G_{j k}^{*}+\sum_{\substack{m=1 \\
m \text { is odd }}}^{+\infty} \mathrm{i}^{m+1} \int_{t_{j}>s_{m}>\cdots>s_{1}>t_{k}} \sum_{\mathfrak{q} \in \mathcal{Q}_{c}\left(t_{j}, \boldsymbol{s}\right)}\right. \\
\left.\quad(-1)^{\#\{\boldsymbol{s}<t\}} \mathcal{L}(\mathfrak{q}) W_{s} G_{I}^{*}\left(t_{j}, s_{m}\right) W_{s} G_{I}^{*}\left(s_{m}, s_{m-1}\right) W_{s} \cdots W_{s} G_{I}^{*}\left(s_{2}, s_{1}\right) W_{s} G_{I}^{*}\left(s_{1}, t_{k}\right) \mathrm{d} s_{1} \cdots \mathrm{d} s_{m}\right],
\end{aligned}
$$


where $G_{I}^{*}(\cdot, \cdot)$ is the interpolated function satisfying $G_{I}^{*}\left(t_{j}, t_{k}\right)=G_{j k}^{*}$ and

$$
G_{I}^{*}\left(t_{j^{\prime}}, t_{k^{\prime}}\right)=G_{j^{\prime} k^{\prime}}^{\Delta t}, \quad \text { for all integers } j^{\prime}, k^{\prime} \text { satisfying } k \leqslant k^{\prime} \leqslant j^{\prime} \leqslant j \text { and }\left(j^{\prime}, k^{\prime}\right) \neq(j, k) .
$$

Again, the same piecewise linear interpolation is adopted.

Note that Heun's method provides more convenient implementation than other second-order Runge-Kutta methods (e.g. mid-point method), since the result of the first stage $\left(G_{j k}^{*}\right)$ is a first-order prediction of $G\left(t_{j}, t_{k}\right)$, so that in the second stage (58), the evaluation of the integral requires no additional interpolation or extrapolation (which is needed if $G_{j k}^{*}$ falls between grid points). When applying (56) and (58), we follow the rules as below:

- When $j=N^{-}, j-1$ is interpreted as $N-1$; when $j=N+1, j-1$ is interpreted as $N^{+}$.

- $\operatorname{sgn}\left(t_{N^{-}}-t\right)=-1$

- The interpolation of $G_{I}$ and $G_{I}^{*}$ should respect such discontinuities. Therefore when $k=N$ or $l=N$, the equation (57) should be interpreted by

$$
\begin{gathered}
\lim _{s \rightarrow t^{ \pm}} G_{I}\left(t_{k}, s\right)=G_{k N^{ \pm}}^{\Delta t}, \quad \lim _{s \rightarrow t^{ \pm}} G_{I}\left(s, t_{l}\right)=G_{N^{ \pm} l}^{\Delta t}, \\
\lim _{s \rightarrow t^{+}} \lim _{\tilde{s} \rightarrow t^{+}} G_{I}(\tilde{s}, s)=\lim _{s \rightarrow t^{-}} \lim _{\tilde{s} \rightarrow t^{-}} G_{I}(s, \tilde{s})=\mathrm{Id}, \quad \lim _{s \rightarrow t^{+}} \lim _{\tilde{s} \rightarrow t^{-}} G_{I}(s, \tilde{s})=O_{s} .
\end{gathered}
$$

The equation (59) should be similarly interpreted. Especially, when $j=N^{+}$, the term $G_{I}\left(t_{N^{+}}, s_{m}\right)$ in the integral should be interpreted as $O_{s} G_{I}\left(t_{N^{-}}, s_{m}\right)$.

The order of computation (55) ensures that all information needed in the two stages has been obtained beforehand.

The above algorithm can be further improved by noticing that the equation (44) has a clear linear part $\mathrm{i} H_{s} G\left(s_{\uparrow}, s_{\mathrm{i}}\right)$. Therefore, when $H_{s}$ is a small matrix, the exponential Runge-Kutta method can be applied. In general, for the initial value problem

$$
\frac{\mathrm{d}}{\mathrm{d} t} u(t)=\mathcal{L} u(t)+f(t, u(t)), \quad t>0
$$

with $\mathcal{L}$ being a linear operator, the Heun's method can be applied to $\mathrm{e}^{-t \mathcal{L}} u$ and we get the numerical scheme

$$
\begin{aligned}
U_{k}^{*} & =\mathrm{e}^{\Delta t \mathcal{L}} U_{k-1}+\Delta t f\left(t_{k-1}, U_{k-1}\right), \\
U_{k} & =\mathrm{e}^{\Delta t \mathcal{L}} U_{k-1}+\frac{1}{2} \Delta t\left[f\left(t_{k-1}, U_{k-1}\right)+\mathrm{e}^{-\Delta t \mathcal{L}} f\left(t_{k}, U_{k}^{*}\right)\right] .
\end{aligned}
$$

In the current case, the operator $\mathcal{L}$ corresponds to $\pm \mathrm{i} H_{s}$. Therefore, when the above scheme is applied, the first term in the second equation is $\mathrm{e}^{ \pm \mathrm{i} \Delta t H_{s}} G_{j-1, k}^{\Delta t}=G_{s}^{(0)}(j \Delta t,(j-1) \Delta t) G_{j-1, k}^{\Delta t}$, which is exactly the first term in the inchworm expansion (23). This is particularly useful if the linear part of the equation is stiff. While for the spin-boson system, which is not stiff, we will be content with the non-exponential integrator described above.

Now we consider the numerical computation of the infinite sums in (56) and (58). The numerical results in 5. show that in the original inchworm method (23), we can truncate the series at $m=M$ for some positive even integer $M$, and obtain results with sufficient quality. In our method, the integer $M$ needs to be odd and we perform the similar truncation by replacing the infinite sums in (56) and (58) with

$$
\sum_{\substack{m=1 \\ m \text { is odd }}}^{M} \mathrm{i}^{m+1} \int_{t_{\mathrm{f}}>s_{m}>\cdots>s_{1}>t_{k}} \sum_{\mathfrak{q} \in \mathcal{Q}_{c}\left(t_{\mathrm{f}}, \boldsymbol{s}\right)} S(\mathfrak{q}, \boldsymbol{s}) \mathrm{d} s_{1} \cdots \mathrm{d} s_{m},
$$

where $S(\mathfrak{q}, s)$ is the summand in (56) or (58), and $t_{\mathrm{f}}$ is the corresponding $t_{j}$ or $t_{j+1}$. For every $m$, the high-dimensional integral over $s$ and the sum over $\mathfrak{q}$ are evaluated using the Monte Carlo method. The 
sampling of $s$ can be done by sampling a uniform distribution in $\left[t_{k}, t_{\mathrm{f}}\right]^{m}$, and then sort the time points. As for $\mathfrak{q}$, we first list out all the elements in $\mathcal{Q}_{c}\left(t_{\mathrm{f}}, s\right)$, and then pick a random one for each sample. Obviously the number of pair sets in $\mathcal{Q}_{c}\left(t_{\mathrm{f}}, \boldsymbol{s}\right)$ depends only on the value of $m$, and it has been given in [53] that this number can be evaluated by

$$
N_{1}=1, \quad N_{m}=\frac{m-1}{2} \sum_{\substack{j=1 \\ j \text { is odd }}}^{m-2} N_{j} N_{m-1-j}
$$

In 54], it is proven that $N_{m}$ grows asymptotically as $m$ !!. In our numerical experiments, we are only concerned about a small $m$, and therefore such a strategy is feasible.

As the end of this section, we will discuss briefly the difference between this numerical method and the original inchworm method based on the Monte Carlo simulation of (23). Both numerical methods are time-stepping methods which can be regarded as a numerical approximation of

$$
G\left(s_{\mathrm{f}}, s_{\mathrm{i}}\right)=G\left(s_{\uparrow}, s_{\mathrm{i}}\right)+\int_{s_{\uparrow}}^{s_{\mathrm{f}}} R H S(s) \mathrm{d} s,
$$

where $R H S(s)$ is the right-hand side of (41) or (42), with $s_{\uparrow}$ replaced by $s$. From the diagrammatic equation (25), one can see that the original inchworm algorithm in principle allows to put an arbitrary number of points between $s_{\uparrow}$ and $s_{\mathrm{f}}$ to evaluate the integral in (61), and all these points are stochastic, which implies that in the time-stepping process, the integral between two time steps is approximated using a Monte Carlo simulation; while in our method, this is replaced by a numerical integration of second-order convergence, which can be expected to be more efficient. One possible benefit of the Monte Carlo integration from $s_{\uparrow}$ to $s_{\mathrm{f}}$ is that when a sufficient number of samples are used, this integral can be evaluated up to arbitrary precision; while in the Runge-Kutta integration, an error of $O\left(\left(s_{f}-s_{\uparrow}\right)^{\alpha}\right)$, where $\alpha$ depends on the order of the method, is always there. However, this does not mean that the numerical error will vanish as the number of samples increases in the original inchworm algorithm, since another part of numerical error comes from the approximation of $R H S(s)$, where the interpolation on the lattice shown in Figure 4 is inevitable in both methods. Such error will only vanish as the grid size tends to zero, but will not vanish as the number of samples tends to infinity. Usually, it is sufficient to match the order of accuracy for evaluating the integral in (61) and the interpolation. This can also be achieved by using another formulation of the original inchworm method (26), and applying Gaussian quadrature to the integral from $s_{\uparrow}$ to $s_{\mathrm{f}}$, which will involve multi-dimensional Gaussian quadrature. The implementation is easier and cheaper by using the Runge-Kutta integration strategy. Another benefit of this new method is that we can draw the samples for $\mathfrak{q}$ more easily. Consider a diagram with four points. The set $\mathcal{Q}_{c}\left(s_{1}, s_{2}, s_{3}, s_{4}\right)$ contains only one set of pairs $\left\{\left(s_{1}, s_{3}\right),\left(s_{2}, s_{4}\right)\right\}$, while the inchworm proper set of pairs has 9 possibilities:

- $\mathcal{Q}_{s_{\uparrow}}\left(s_{1}, s_{2}, s_{3}, s_{4}\right)=\left\{\left\{\left(s_{1}, s_{3}\right),\left(s_{2}, s_{4}\right)\right\}\right\}$ if $s_{3}<s_{\uparrow} \leqslant s_{4}$

- $\mathcal{Q}_{s_{\uparrow}}\left(s_{1}, s_{2}, s_{3}, s_{4}\right)=\left\{\left\{\left(s_{1}, s_{3}\right),\left(s_{2}, s_{4}\right)\right\},\left\{\left(s_{1}, s_{4}\right),\left(s_{2}, s_{3}\right)\right\}\right\}$ if $s_{2}<s_{\uparrow} \leqslant s_{3}$;

- $\mathcal{Q}_{s_{\uparrow}}\left(s_{1}, s_{2}, s_{3}, s_{4}\right)=\left\{\left\{\left(s_{1}, s_{3}\right),\left(s_{2}, s_{4}\right)\right\},\left\{\left(s_{1}, s_{4}\right),\left(s_{2}, s_{3}\right)\right\},\left\{\left(s_{1}, s_{2}\right),\left(s_{3}, s_{4}\right)\right\}\right\}$ if $s_{1}<s_{\uparrow} \leqslant s_{2}$;

- $\mathcal{Q}_{s_{\uparrow}}\left(s_{1}, s_{2}, s_{3}, s_{4}\right)=\left\{\left\{\left(s_{1}, s_{3}\right),\left(s_{2}, s_{4}\right)\right\},\left\{\left(s_{1}, s_{4}\right),\left(s_{2}, s_{3}\right)\right\},\left\{\left(s_{1}, s_{2}\right),\left(s_{3}, s_{4}\right)\right\}\right\}$ if $s_{\uparrow} \leqslant s_{1}$.

For the more time points, finding out all the inchworm proper pair sets is even harder than just finding out $\mathcal{Q}_{c}(s)$. This also makes the implementation of Runge-Kutta method cheaper than Gaussian quadrature.

Remark 5. As mentioned in Remark 2, we focus on the bosonic setting in this work. For fermionic settings, the number of inchworm proper diagrams also grows asymptotically as $O\left(\left(\frac{m+1}{2}\right) !\right)$, as indicated in [2]. On the other hand, in fermionic settings, by a more sophisticated combinatoric technique called inclusionexclusion principle [2], the number of diagrams to be summed can be reduced intrinsically to $O\left(m^{3} \beta^{m}\right)$ for a constant $\beta<2$. This is to some extent in the similar spirit of the reduced cost of diagrammatic summations achieved by the determinant diagrammatic Monte Carlo method [51] for fermionic systems. 


\section{Numerical experiments}

In our numerical experiments, we consider the spin-Boson model with a bath with Ohmic spectral density, for which the frequency $\omega_{l}$ are distributed in $\left[0, \omega_{\max }\right]$ as introduced in [37]:

$$
\omega_{l}=\omega_{c} \ln \left(1-\frac{l}{L}\left[1-\exp \left(\omega_{\max } / \omega_{c}\right)\right]\right), \quad l=1, \cdots, L,
$$

where $\omega_{c}$ is the primary frequency to be specified later. The coupling intensity $c_{l}$ is

$$
c_{l}=\omega_{l} \sqrt{\frac{\xi \omega_{c}}{L}\left[1-\exp \left(\omega_{\max } / \omega_{c}\right)\right]}, \quad l=1, \cdots, L,
$$

with $\xi$ being the Kondo parameter. To compare our results with reference solutions, we adopt the parameters provided in [29] where $L=200$ and $\beta=5 \Delta^{-1}$. Different settings of bias, coupling intensity and nonadiabaticity will be considered in our experiments. In all the numerical tests, the maximum frequency $\omega_{\max }$ is set to be $4 \omega_{c}$, and the time step is chosen to be $\Delta t=0.1$ if not otherwise specified. Numerical results obtained by the QuAPI method [39, 40] will be used as reference solutions.

\subsection{Experiments with changing bias}

We first choose $\omega_{c}=2.5 \Delta$ and $\xi=0.2$, for which the amplitude of the bath correlation function $B(\cdot, \cdot)$ is plotted in Figure 5. In our implementation, we precompute the values of $B(\cdot, \cdot)$ up to a very high precision on a very fine grid, and in the inchworm algorithm, we retrieve its value by linear interpolation when necessary. The numerical results for $\epsilon=0, \Delta$ and $2 \Delta$ are given in Figure 6. It turns out that $M=3$ in (60) can already provide satisfying numerical results up to time $t=5 \Delta^{-1}$, and the general behavior of the observable has been well captured by the results of $M=1$.

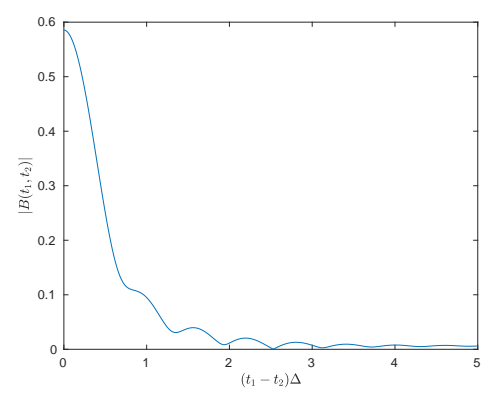

Figure 5: The amplitude of the bath correlation function $B(\cdot, \cdot)$

The order of convergence of our numerical method is also verified using the test case with $\epsilon=0$. In general, the stochastic error and the "deterministic error" caused by Runge-Kutta and interpolation cannot be separated. In order to cast off the stochastic error, we only consider the truncation $M=1$, for which the sum (60) contains only a one-dimensional integral, and thus can be evaluated by the composite mid-point rule. As a result, the whole scheme is deterministic and the order of convergence is still expected to be $O\left(\Delta t^{2}\right)$. The reference solution is obtained by choosing $\Delta t=1 / 320 \Delta^{-1}$, and the numerical error of $\left\langle\hat{\sigma}_{z}(t)\right\rangle$ is tabulated in Table 1, which clearly shows the numerical error is second order of $\Delta t$.

\subsection{Experiments with changing coupling intensity}

Now we fix the values of $\omega_{c}$ and $\epsilon$ to be $2.5 \Delta$ and $\Delta$ respectively, and consider the coupling intensity $\xi=0.1$ and $\xi=0.2$. It can be expected that the convergence of the inchworm series gets slower when $\xi$ increases, as is confirmed in our numerical tests shown in Figure 7 . In spite of this, very good matching with the QuAPI results can still be obtained using $M=1$ for both parameters, and further improvement is indeed achieved by using $M=3$. 

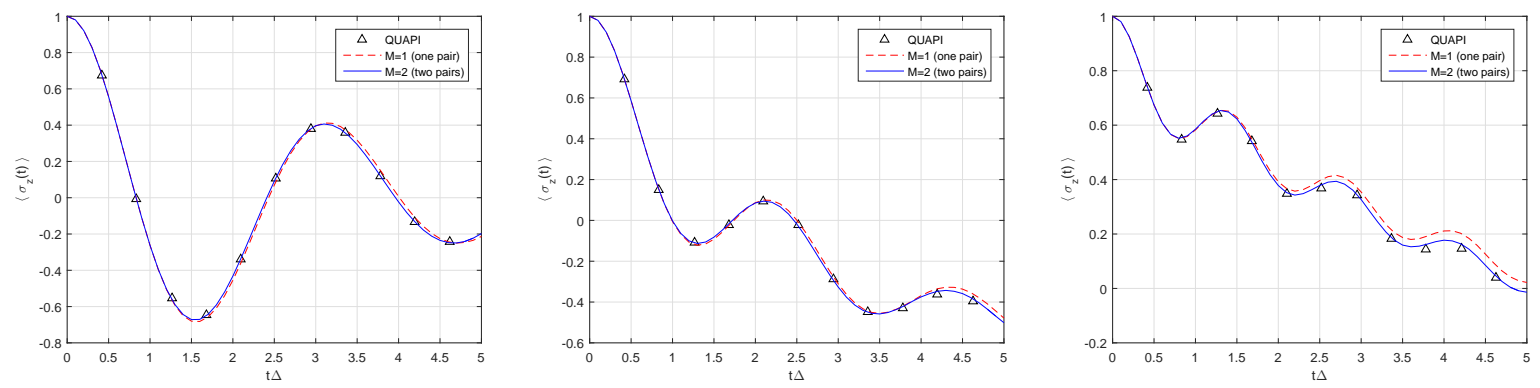

Figure 6: Evolution of $\left\langle\sigma_{z}(t)\right\rangle$ under different settings of the electronic bias (from left to right: $\epsilon=0, \epsilon=\Delta$ and $\epsilon=2 \Delta$ ), with other parameters $\omega_{c}=2.5 \Delta$ and $\xi=0.2$. QuAPI results are plotted as a reference.

\begin{tabular}{c||cc|cc|cc|cc}
\hline$h$ & error $(t=0.5)$ & order & error $(t=1)$ & order & error $(t=1.5)$ & order & error $(t=2)$ & order \\
\hline $1 / 10$ & 0.0014 & - & 0.0022 & - & 0.0014 & - & 0.0067 & - \\
$1 / 20$ & 0.0004 & 1.9740 & 0.0005 & 2.0537 & 0.0004 & 1.8855 & 0.0017 & 1.9910 \\
$1 / 30$ & $0.1620 \times 10^{-3}$ & 1.9961 & $0.2343 \times 10^{-3}$ & 2.0307 & $0.1722 \times 10^{-3}$ & 1.9632 & $0.7490 \times 10^{-3}$ & 2.0064 \\
$1 / 40$ & $0.0909 \times 10^{-3}$ & 2.0087 & $0.1305 \times 10^{-3}$ & 2.0344 & $0.0970 \times 10^{-3}$ & 1.9941 & $0.4189 \times 10^{-3}$ & 2.0204 \\
$1 / 50$ & $0.0578 \times 10^{-3}$ & 2.0299 & $0.0826 \times 10^{-3}$ & 2.0470 & $0.0618 \times 10^{-3}$ & 2.0188 & $0.2658 \times 10^{-3}$ & 2.0376 \\
$1 / 60$ & $0.0398 \times 10^{-3}$ & 2.0505 & $0.0567 \times 10^{-3}$ & 2.0657 & $0.0426 \times 10^{-3}$ & 2.0422 & $0.1826 \times 10^{-3}$ & 2.0587 \\
\hline
\end{tabular}

Table 1: Numerical error of $\left\langle\hat{\sigma}_{z}(t)\right\rangle$ and the order of accuracy

As a reference, the numerical results for a direct summation of the truncated Dyson series

$$
G\left(s_{\mathrm{f}}, s_{\mathrm{i}}\right) \approx \sum_{\substack{m=0 \\ m \text { is even }}}^{M} \int_{s_{\mathrm{f}}>s_{m}>\cdots>s_{1}>s_{\mathrm{i}}} \sum_{\mathfrak{q} \in \mathcal{Q}(\boldsymbol{s})}(-1)^{\#\{\boldsymbol{s}<t\}_{\mathrm{i}}{ }^{m}} \mathcal{U}^{(0)}\left(s_{\mathrm{f}}, \boldsymbol{s}, s_{\mathrm{i}}\right) \mathcal{L}(\mathfrak{q}) \mathrm{d} s_{1} \cdots \mathrm{d} s_{m},
$$

are also provided in Figure 7 with label "bare dQMC". The results show that the convergence of the Dyson series (19) is much slower than the inchworm series, and therefore require a much larger $M$ to get the same quality of the solutions for large $t$.
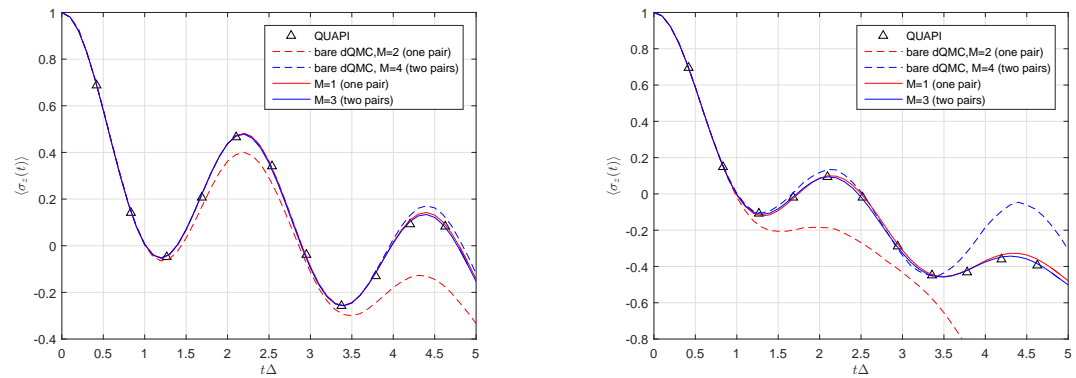

Figure 7: Evolution of $\left\langle\sigma_{z}(t)\right\rangle$ under different settings of the coupling intensity (left: $\xi=0.1$, right: $\xi=0.2$ ), with other parameters $\omega_{c}=2.5 \Delta$ and $\epsilon=\Delta$. QuAPI results are plotted as a reference.

\subsection{Experiments with changing nonadiabaticity}

To show the role of the parameter $\omega_{c}$, we fix $\xi$ to be 0.4 and $\epsilon$ to be $\Delta$, and consider the cases $\omega_{c}=0.25 \Delta$ and $\omega_{c}=2.5 \Delta$. Since the upper bound of $\left|B\left(\tau_{1}, \tau_{2}\right)\right|$ in (52) gets larger when $\omega_{c}$ increases, we can expect slower convergence in terms of $M$ for larger $\omega_{c}$. The evolution of the observable is plotted in Figure 8 , In the right figure, due to the large value of both $\xi$ and $\omega_{c}$, even when $M=3$ is used, some discrepancy between our results and QuAPI can still be observed. 

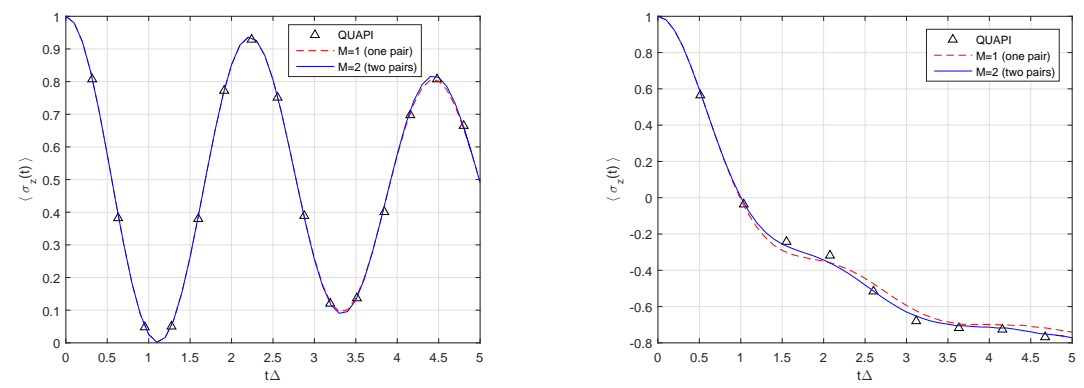

Figure 8: Evolution of $\left\langle\sigma_{z}(t)\right\rangle$ under different settings of the nonadiabaticity (left: $\omega_{c}=0.25 \Delta$, right: $\omega_{c}=2.5 \Delta$ ), with other parameters $\xi=0.4$ and $\epsilon=\Delta$. QuAPI results are plotted as a reference.

\section{Summary}

We have studied the inchworm Monte Carlo method introduced in [4, 10], and proven rigorously that the method converges to the solution of the open quantum system whose bath configuration satisfies Wick's theorem. By assuming the iterative step length to be infinitesimal, we have derived a new continuous model for the open quantum system, and have proposed an improvement of the inchworm method to achieve better numerical efficiency and simpler implementation. Such a framework is not restricted to the specific case considered in this paper. In fact, the inchworm method has been applied to a number of cases beyond the settings of this paper, including

- The fermionic bath [1, 10], which needs a revision of the definition of $\mathcal{L}(\mathfrak{q})$ (16);

- Full Keldysh contour 1], which requires to augment Figure 1 with an imaginary time (equilibrium) part of the contour;

- Two-time observables such as Green's functions [1], which adds some additional restrictions on the admissible diagrams;

- Non-unitary dynamics in the existence of an auxiliary counting field [50], in which a non-Hermitian time-dependent Hermitian is introduced.

These extensions may complicate the mathematical formulation, while the general ideas can be extended without much difficulty, and it can be expected that new numerical methods based on integral-differential equation formalism can be derived accordingly. For the new numerical method, the stochastic error and the deterministic error are coupled together, and one needs to apply numerical analysis to find optimal combinations of parameters. This will be an interesting future direction.

Possible applications of this method to dimension reduction of classical systems are also interesting. One interesting possibility is high-dimensional Langevin dynamics, especially when the system has a conditional Gaussian structure, such as that considered in [6]. Due to the similarity between the Fokker-Planck operator and the Hamiltonian operator, the inchworm method can be applied under certain conditions. More general problems may require more sophisticated resummation techniques. These extensions are left for future works.

\section{Acknowledgements}

The work of Zhenning Cai is supported by National University of Singapore Startup Fund under Grant No. R-146-000-241-133. The work of Jianfeng Lu is supported in part by National Science Foundation under grant DMS-1454939. This collaboration is also supported by National Science Foundation under grant RNMS-1107444 (KI-Net). 


\section{References}

[1] A. E. Antipov, Q. Dong, J. Kleinhenz, G. Cohen, and E. Gull. Currents and Green's functions of impurities out of equilibrium: Results from inchworm quantum Monte Carlo. Phys. Rev. B, 95:085144, 2017.

[2] A. Boag, E. Gull, and G. Cohen. Inclusion-exclusion principle for many-body diagrammatics. Phys. Rev. B, 98:115152, 2018.

[3] H. Breuer and F. Petruccione. The Theory of Open Quantum Systems. Oxford University Press, 2007.

[4] H.-T. Chen, G. Cohen, and D. R. Reichman. Inchworm Monte Carlo for exact non-adiabatic dynamics. I. Theory and algorithms. J. Chem. Phys., 146:054105, 2017.

[5] H.-T. Chen, G. Cohen, and D. R. Reichman. Inchworm Monte Carlo for exact non-adiabatic dynamics. II. Benchmarks and comparison with established methods. J. Chem. Phys., 146:054106, 2017.

[6] N. Chen and A. J. Majda. Beating the curse of dimension with accurate statistics for the Fokker-Planck equation in complex turbulent systems. Proc. Nat. Acad. Sci. USA, 114:12864-12869, 2017.

[7] A. J. Chorin and O. H. Hald. Stochastic Tools in Mathematics and Science. Springer, New York, 3rd edition, 2013.

[8] A. J. Chorin, O. H. Hald, and R. Kupferman. Optimal prediction and the Mori-Zwanzig representation of irreversible processes. Proc. Natl. Acad. Sci. USA, 97:2968-2973, 2000.

[9] A. J. Chorin and Fei Lu. Discrete approach to stochastic parametrization and dimension reduction in nonlinear dynamics. Proc. Natl. Acad. Sci. USA, 112:9804-9809, 2015.

[10] G. Cohen, E. Gull, D. R. Reichman, and A. J. Millis. Taming the dynamical sign problem in real-time evolution of quantum many-body problems. Phys. Rev. Lett., 115(26):266802, 2015.

[11] E. Darve, J. Solomon, and A. Kia. Computing generalized Langevin equations and generalized Fokker-Planck equations. Proc. Natl. Acad. Sci. USA, 106:10884-10889, 2009.

[12] E. B. Davies. Markovian master equations. Commun. Math. Phys., 39(2):91-110, 1974.

[13] E. B. Davies. Markovian master equations. II. Math. Ann., 219(2):147-158, 1976.

[14] Q. Dong, I. Krivenko, J. Kleinhenz, A. E. Antipov, G. Cohen, and E. Gull. Quantum monte carlo solution of the dynamical mean field equations in real time. Phys. Rev. B, 96:155126, 2017.

[15] C. Duan, Z. Tang, J. Cao, and J. Wu. Zero-temperature localization in a sub-ohmic spin-boson model investigated by an extended hierarchy equation of motion. Phys. Rev. B, 95(21):214308, 2017.

[16] F. J. Dyson. The radiation theories of Tomonaga, Schwinger, and Feynman. Phys. Rev., 75(3):486-502, 1949.

[17] Weinan E. Principles of Multiscale Modeling. Cambridge University Press, 2011.

[18] M. Esposito, K. Lindenberg, and C. Van den Broeck. Entropy production as correlation between system and reservoir. New J. Phys., 12(1):013013, 2010.

[19] D. Evans and G. Morris. Statistical Mechanics of Nonequilibrium Liquids. Academic, 1990.

[20] E. Garnier, N. Adams, and P. Sagaut. Large Eddy Simulation for Compressible Flows. Springer, 2009.

[21] E. Gull, D. R. Reichman, and A. J. Millis. Bold-line diagrammatic Monte Carlo method: General formulation and application to expansion around the noncrossing approximation. Phys. Rev. B, 82(7):075109, 2010.

[22] E. Gull, D. R. Reichman, and A. J. Millis. Numerically exact long-time behavior of nonequilibrium quantum impurity models. Phys. Rev. B, 84(8):085134, 2011.

[23] Emanuel Gull, Andrew J. Millis, Alexander I. Lichtenstein, Alexey N. Rubtsov, Matthias Troyer, and Philipp Werner. Continuous-time Monte Carlo methods for quantum impurity models. Rev. Mod. Phys., 83:349-404, 2011.

[24] R. J. Henery. The generalized Langevin equation. J. Phys. A: Gen. Phys., 5:1312, 1972.

[25] Carmen Hijön, Pep Espaöl, Eric Vanden-Eijnden, and Rafael Delgado-Buscalioni. Mori-Zwanzig formalism as a practical computational tool. Faraday Discuss., 144:301-322, 2010.

[26] L. P. Kadanoff and G. Baym. Quantum Statistical Mechanics: Green's Function Methods in Equilibrium and NonEquilibrium Problems. W.A. Benjamin, 1962.

[27] E. Kalnay. Atmospheric Modeling, Data Assimilation, and Predictability. Cambridge University Press, 2003.

[28] L. V. Keldysh. Diagram technique for nonequilibrium processes. Sov. Phys. JETP, 20(4):1018-1026, 1965.

[29] A. Kelly and T. E. Markland. Efficient and accurate surface hopping for long time nonadiabatic quantum dynamics. J. Chem. Phys., 139(1):014104, 2013.

[30] D. Mac Kernan, G. Ciccotti, and R. Kapral. Surface-hopping dynamics of a spin-boson system. J. Chem. Phys., 116(6):2346-2353, 2002.

[31] H. Lei, N. A. Baker, and X. Li. Data-driven parameterization of the generalized Langevin equation. Proc. Natl. Acad. Sci. USA, 113:14183-14188, 2016.

[32] C. D. Levermore. Moment closure hierarchies for kinetic theories. J. Stat. Phys., 83(5-6):1021-1065, 1996.

[33] Yingzhou Li and Jianfeng Lu. Bold diagrammatic Monte Carlo in the lens of stochastic iterative methods. Trans. Math. Appl., 3:1-17, 2019.

[34] K. Lindenberg and B. J. West. The Nonequilibrium Statistical Mechanics of Open and Closed Systems. Wiley, 1990.

[35] D. E. Makarov and N. Makri. Path integrals for dissipative systems by tensor multiplication. Condensed phase quantum dynamics for arbitrarily long time. Chem. Phys. Lett., 221(5-6):482-491, 1994.

[36] N. Makri. Numerical path integral techniques for long time dynamics of quantum dissipative systems. J. Math. Phys., 36(5):2430-2457, 1995.

[37] N. Makri. The linear response approximation and its lowest order corrections: An influence functional approach. J. Phys. Chem. B, 103(15):2823-2829, 1999.

[38] N. Makri. Iterative blip-summed path integral for quantum dynamics in strongly dissipative environments. J. Chem. Phys., 146(13):134101, 2017. 
[39] N. Makri and D. E. Makarov. Tensor propagator for iterative quantum time evolution of reduced density matrices. I. theory. J. Chem. Phys., 102(11):4600-4610, 1995.

[40] N. Makri and D. E. Makarov. Tensor propagator for iterative quantum time evolution of reduced density matrices. II. numerical methodology. J. Chem. Phys., 102(11):4611-4618, 1995.

[41] N. Makri, E. Sim, D. E. Makarov, and M. Topaler. Long-time quantum simulation of the primary charge separation in bacterial photosynthesis. Proc. Natl. Acad. Sci., 93(9):3926-3931, 1996.

[42] C. Meier and D. J. Tannor. Non-Markovian evolution of the density operator in the presence of strong laser fields. J. Chem. Phys., 111:3365, 1999 .

[43] A. Montoya-Castillo and D. R. Reichman. Approximate but accurate quantum dynamics from the Mori formalism: I. Nonequilibrium dynamics. J. Chem. Phys., 144:184104, 2016.

[44] H. Mori. Transport, collective motion, and Brownian motion. Prog. Theor. Phys., 33:423-455, 1965.

[45] S. Nakajima. On quantum theory of transport phenomena. Prog. Theo. Phys., 20(6):948-959, 1958.

[46] F. Nicoud. Using singular values to build a subgrid-scale model for large eddy simulations. Phys. Fluids, 23(8):085106, 2011.

[47] M. A. Nielsen and I. L. Chuang. Quantum Computation and Quantum Information: 10th Anniversary Edition. Cambridge University Press, 2010.

[48] M. B. Plenio and S. F. Huegla. Entangled light from white noise. Phys. Rev. Lett., 88:197901, 2002.

[49] N. Prokof'ev and B. Svistunov. Bold diagrammatic Monte Carlo technique: When the sign problem is welcome. Phys. Rev. Lett., 99(25):250201, 2007.

[50] M. Ridley, V. N. Singh, E. Gull, and G. Cohen. Numerically exact full counting statistics of the nonequilibrium Anderson impurity model. Phys. Rev. B, 97(11):115109, 2018.

[51] R. Rossi. Determinant diagrammatic Monte Carlo algorithm in the thermodynamic limit. Phys. Rev. Lett., 119:045701, 2017.

[52] Q. Shi and E. Geva. A new approach to calculating the memory kernel of the generalized quantum master equation for an arbitrary system-bath coupling. J. Chem. Phys., 119:12063, 2003.

[53] P. R. Stein. On a class of linked diagrams, I. Enumeration. J. Comb. Theory Ser. A, 24:357-366, 1978.

[54] P. R. Stein. On a class of linked diagrams, II. Asymptotics. Discrete Math., 21:309-318, 1978.

[55] J. T. Stockburger and H. Grabert. Exact c-number representation of non-Markovian quantum dissipation. Phys. Rev. Lett., 88(17):170407, 2002.

[56] J. Strümpfer and K. Schulten. Open quantum dynamics calculations with the hierarchy equations of motion on parallel computers. J. Chem. Theory Comput., 8(8):2808-2816, 2012.

[57] K.-A. Suominen. Open Quantum Systems and Decoherence, pages 247-282. Springer International Publishing, 2014.

[58] M. Torrilhon. Modeling nonequilibrium gas flow based on moment equations. Ann. Rev. Fluid Mech., 48:429-458, 2016.

[59] H. Wang. Basis set approach to the quantum dissipative dynamics: Application of the multiconfiguration time-dependent Hartree method to the spin-boson problem. J. Chem. Phys., 113(22):9948-9956, 2000.

[60] P. Werner, T. Oka, and A. J. Millis. Diagrammatic Monte Carlo simulation of nonequilibrium systems. Phys. Rev., 79(3):035320, 2009.

[61] E. Zwanzig. Ensemble method in the theory of irreversibility. J. Chem. Phys., 33(5):1338-1341, 1960.

[62] R. Zwanzig. Memory effects in irreversible thermodynamics. Phys. Rev., 124:983-992, 1961.

[63] R. Zwanzig. Nonlinear generalized Langevin equations. J. Stat. Phys., 9:215-220, 1973. 